\section{Maternal obesity and offspring body composition by indirect methods: a systematic review and meta-analysis}

\author{
Obesidad maternal y composición corporal de los \\ hijos por métodos indirectos: revisión sistemática \\ y metaanálisis
}

\author{
Obesidade materna e composição corporal dos \\ filhos por métodos indiretos: revisão sistemática \\ e meta-análises
}

\author{
1 Faculdade de Medicina, \\ Universidade Federal de \\ Pelotas, Pelotas, Brasil. \\ 2 Faculdade de Medicina, \\ Universidade de São Paulo, \\ São Paulo, Brasil. \\ Correspondence \\ H. Castillo-Laura \\ Departamento de Medicina \\ Preventiva, Faculdade de \\ Medicina, Universidade \\ Federal de Pelotas. \\ Rua Marechal Deodoro 1160, \\ 3 o andar, C.P. 464, Pelotas, RS \\ 96020-220, Brasil. \\ helenclaura@gmail.com
}

\begin{abstract}
This study reviewed the evidence that assessed the association between maternal pre-pregnancy body mass index (BMI) and/or gestational weight gain and offspring body composition in childhood. A systematic review was conducted. Cohort studies, case-control studies and randomized controlled trials measuring offspring body composition by indirect methods were included. Meta-analyses of the effect of pre-pregnancy BMI on offspring fat-free mass, body fat percent, and fat mass were conducted through random-effects models. 20 studies were included, most of which reported a positive association of pre-pregnancy BMI with offspring body fat. Standardized mean differences in body fat percent, fat mass and fat-free mass between infants of women with normal pre-pregnancy BMI and those of overweight/obese women were 0.31 percent points (95\%CI: $0.19 ; 0.42), 0.38 \mathrm{~kg}$ (95\%CI: 0.26 ; 0.50), and $0.18 \mathrm{~kg}$ (95\%CI: -0.07; 0.42), respectively. Evidence so far suggests that pre-pregnancy maternal overweight is associated with higher offspring adiposity.
\end{abstract}

Obesity; Pregnancy; Body Composition; Adiposity
Helen Castillo-Laura 1

Iná S. Santos 1

Lenice C. M. Quadros 1

Alicia Matijasevich ${ }^{2}$

\section{Resumen}

Esta revisión evaluó la asociación entre el índice de masa corporal (IMC) pregestacional y/o el aumento de peso gestacional con la composición corporal de los hijos en la infancia. Se realizó una revisión sistemática. Se incluyeron estudios de cohorte, caso-control y ensayos randomizados, que determinaron la composición corporal de los hijos mediante métodos indirectos. Se realizaron metaanálisis del efecto del IMC pregestacional sobre la masa libre de grasa, porcentaje de grasa corporal y masa de grasa. Se incluyeron 18 estudios, la mayoría de los cuales reportó asociación positiva entre el IMC materno pregestacional y la adiposidad de los hijos. Las diferencias de medias padronizadas de porcentaje de grasa corporal, masa de grasa y masa libre de grasa entre hijos de mujeres con IMC pregestacional normal, en comparación con los de mujeres con sobrepeso/obesidad fueron: 0,31 puntos porcentuales (IC95\%: 0,19; 0,42), 0,38kg (IC95\%: 0,26; 0,50) y 0,18kg (IC95\%: -0,07; 0,42), respectivamente. Esta evidencia sugiere que el sobrepeso materno pregestacional se asocia con una elevada adiposidad en sus hijos.

Obesidad; Embarazo; Composición Corporal; Adiposidad 


\section{Introduction}

According to observational studies, carried out in high income countries, maternal lifestyle, dietary habits, physical activity and work hours could influence offspring size at birth, and weight development in childhood 1,2,3. Pre-pregnancy and gestational maternal body composition may impact offspring body fat mass at birth, in childhood and in adolescence, and may also predict the risk of obesity in adulthood 4,5,6,7,8. The increase in the prevalence of maternal obesity in recent decades 9,10 has occurred in parallel with the increment of large for gestational age newborns 11,12,13 and an increased incidence of childhood obesity 14 . Female offspring of obese women are most likely to be obese predisposing their children to obesity, insulin resistance and diabetes $6,15,16$. Several studies have shown that a high increment in maternal gestational weight gain associated with increased offspring body mass index (BMI) 17,18,19 whereas other studies have reported no such association 20,21. In consequence, across a number of generations a potential intergenerational cycle of increased risk of obesity and metabolic sequelae in the offspring of obese mothers is expected, with important implications for public health policies $4,11,12$.

In investigating the relationship of high maternal pre-pregnancy BMI or gestational weight gain and increased offspring body composition at various ages, the majority of studies have employed double indirect methods (bioelectrical impedance and anthropometric measurements) $7,8,21,22$ that use a two-component model, dividing body weight into fat mass and fat free mass 23 . Recently indirect measuring methods including air displacement plethysmography (ADP), dual energy X-ray absorptiometry (DEXA), total body water (TBW), and body electrical conductivity (TOBEC) have been introduced to the assessment of body composition. These indirect measuring methods are based on a three- or four-compartment model, providing accurate measurements of body fat, lean mass, bone mass, and TBW 23,24.

This review aimed to: (a) identify studies that investigated the relationship between maternal pre-pregnancy BMI and/or gestational weight gain and offspring fat mass, lean mass, fat-free mass, fat mass index, fat free mass index, lean mass index, body fat percent as measured by indirect methods; and (b) calculate a pooled measure of the strength of the association.

\section{Methods}

This systematic review included studies published between the beginning of the indexing by databases until August 2015. The reporting guidance included in the preferred reporting items for systematic reviews and meta-analyses (PRISMA) statement followed 25.

a) Eligibility criteria: prospective or retrospective cohorts, case-control studies, cross-sectional and randomized controlled trials that evaluated the association between maternal pre-pregnancy BMI and/or gestational weight gain with offspring body composition by indirect methods were eligible for the review. Participants included mother-offspring pairs with the exception of outcomes measured in adolescents and adult populations. Measurements of the outcomes were obtained by DEXA, ADP or TOBEC equipments (indirect methods).

b) Search strategy: a search in the electronic MEDLINE, LILACS and SciELO databases and cited references of the select articles was performed. The search was restricted to publications in English, French, Portuguese, Spanish and Italian and original studies in humans. The Medical Subject Headings (MeSH) descriptors used were: " maternal weight, over-nutrition, anthropometry, under-nutrition, body mass index, size, obesity, nutritional status"; OR "prenatal bodymass index, obesity, weight, and height”; OR "pre-pregnancy weight, obesity, body mass index"; OR "pregnancy weight gain"; OR "gestational weight gain" combined with "body composition", "body fat", "body fat percentage", "fat mass", "lean mass”, "fat mass index", "lean mass index", "fat free mass", "adiposity", "ADP", "TOBEC" and "DEXA".

c) Data collection process: the study selection was conducted in duplicate by two independent evaluators in order to screen articles which were stored in EndNote (http://www.endnote.com; Thomson Reuters, USA). The screening stages were: (1) reading study titles for potential inclusion; (2) reading the abstracts of study titles with potential inclusion and selected the full-text articles; (3) full-text articles screening; and (4) assessment of selected articles. Disagreements among the evaluators regarding study eligibility following abstract and full-text review were solved by consensus.

The criteria for selecting articles to be fully reviewed included: (1) prospective or retrospective cohort or case-control design; (2) studies with maternal pre-pregnancy BMI and/or gestational weight gain as the main maternal exposures; and (3) studies with at least one of the following outcomes: lean mass, fat mass, fat-free mass, body 
fat percent, lean mass index (LMI), fat mass index (FMI), and high adiposity in children.

d) Data extraction: for data extraction, the two independent evaluators were responsible for collecting primary data from the selected studies, which were stored in Excel (Microsoft Corp., USA). Information related to study characteristics, such as year, city/country, design, inclusion and exclusion criteria, sample size or number of participants, maternal exposure, age of outcome measurement, indirect method of outcome measurement, and analysis adjustment variables was gathered. Outcome data was extracted independently of the type of the measure of effect presented by the authors (mean, relative risk, odd ratio, etc.). Disagreements among the evaluators regarding data items were solved by consensus.

e) Risk of bias in individual studies: to assess the quality of the randomized control trials the 27-items Downs and Black scale was employed 26 . 19 items of the original scale were used to assess the quality of observational studies: type of study, hypothesis/aim, outcomes, exposures, participant selection, loss of follow-up, random variability, statistical method, probability values, source population, study sample, bias, data dredging, lengths of follow-up, precision, confounding and power. According to Downs \& Black 26, randomized controlled trials could achieve a score ranging from 0 to 27 points, and the observational studies 0 to 20 points. Study quality was determined by the higher score.

f) Summary measures: for meta-analysis the data extracted included the mean (M), standard deviation (SD) or standard error of the mean (SEM), and the sample size (N) for two groups of women: underweight/normal-weight and overweight/obese, for each continuous outcome (body fat percent, fat mass and fat-free mass). The aggregated effect of pre-pregnancy BMI was estimated by calculating the standardized mean differences. The random-effects model for fatfree mass (because heterogeneity between studies was detected) and the fixed-effects model for body fat percent and fat mass, and the corresponding 95\% confidence intervals (95\%CI) were estimated. The aggregated results for these seven studies are presented in forest plot graphics. Meta-regression and subgroup analysis were conducted to examine the impact of moderator variables on fat-free mass standardized mean difference (SMD). Subgroup analysis was considered and the subsets were study sample, outcome age measurement, study design, indirect method to outcome measurement and confounding control. Stata version 12.0 (StataCorp LP, College Station, USA) was used for data analyses. g) Risk of bias across studies: statistical heterogeneity among studies was assessed with the Q and $\mathrm{I}^{2}$ statistics tests. Publication bias was investigated with Begg's funnel plot and was further assessed using the Egger's linear regression test method.

\section{Results}

A total of 6,561 articles were initially retrieved: 3,972 duplicate records were removed and 2,589 records screened. Studies on animals, basic sciences, and chronic diseases $(n=2,178)$ were excluded after a first reading of titles, resulting in 411 abstracts to be analyzed. For 349 abstracts in the review, the exclusion criteria considered were: studies that investigated other distal, intermediate or proximal determinants of offspring's body composition but not maternal prepregnancy BMI or gestational weight gain $(\mathrm{n}=$ 72); studies investigating offspring growth patterns using only anthropometric methods ( $\mathrm{n}=$ 99); studies investigating the feeding patterns of pregnant women, parents and children $(n=75)$; studies analyzing specific groups of women including mothers with gestational diabetes $(\mathrm{n}=$ 62); studies investigating fetal programming ( $\mathrm{n}=$ 23); validation of body composition by indirect methods $(n=18)$. Only studies that were clearly irrelevant were excluded at the first stage. The full text of identified articles including those where there were disagreements in the initial title/abstract scanning, were reviewed to ensure that the inclusion criteria were met.

Of the 62 remaining articles, 42 were excluded for the following reasons: studies that investigated maternal exposure after pregnancy $(n=5)$; outcome evaluated by doubly indirect methods ( $\mathrm{n}=33$ ); outcome in adulthood $(\mathrm{n}=2)$; maternal height and tricipital skinfold thickness exposures $(n=1)$; exposure evaluated by indirect methods $(n=1)$. The cited references of the 20 articles selected were reviewed however no additional publications were found. Figure 1 shows the number of articles retrieved, then excluded at each stage of the review process and the corresponding reasons for their exclusion.

Among the 20 articles that met the inclusion criteria, six included both exposures 6,27,28,29,30,31. The average score of the selected articles was 15.4, with a minimum of 13 and a maximum of 18. Table 1 displays a description of the selected studies: first author's name; city and country where the study was conducted; study design; number (n) of study participants; eligibility criteria; maternal exposures; offspring's age when the outcome was assessed; indirect methods used 
Flowchart for the process of selection of articles on maternal pre-pregnancy body mass index (BMI) and gestational weight gain offspring body composition in childhood, 2015.

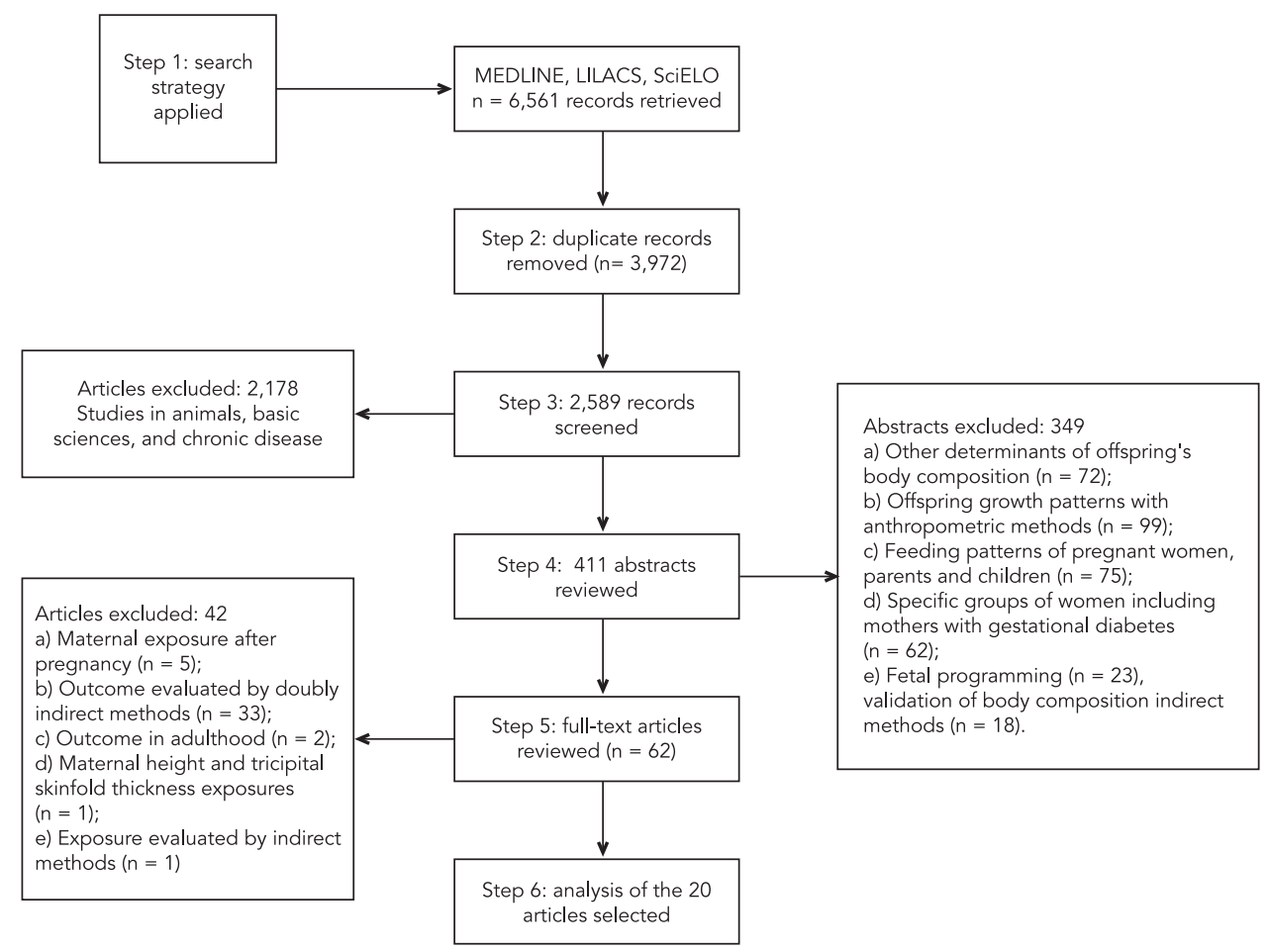

for assessment of body composition of offspring; and confounding variables used in the statistical analyses according to maternal exposures.

\section{Studies that assessed the effect of maternal pre-pregnancy BMI}

In 16 of the 20 articles selected, maternal prepregnancy BMI was the main exposure investigated. Ten studies were conducted in the United States 5,6,29,30,32,33,34,35,36,37, two in the United Kingdom 28,38, two in Denmark 32,39, one in Australia 27, one in Brazil 31, and one in Portugal 40 (Table 1). The number of participants in the cohorts ranged from 7834 to 3,263 38 . Although these studies applied different inclusion and exclusion criteria, all consistently excluded premature newborns, those with congenital abnormalities, offspring of mothers with a history of pregestational or gestational diabetes, and children with chronic diseases. With the exception of five studies $27,29,31,35,36$, all other studies included only Caucasian participants.
All studies except for three 28,30,37, stratified maternal pre-pregnancy BMI to establish maternal overweight and/or obesity. Eight studies measured body composition in newborns $5,6,27,29,32,33,37,40$, whilst all other studies measured body composition in childhood (2-11 years of age). The methods for the assessment of body composition included DEXA in eight studies $28,30,32,34,35,36,38,39$, ADP in seven 5,6,27,31,33,37,38 and TOBEC in one 29 (Table 2).

\section{- Pre-pregnancy BMI and offspring adiposity}

Table 2 summarizes the main results of the 16 studies reviewed. 13 reported fat mass 5,6,29,30, $31,32,33,34,35,37,38,39,40$ and two FMI 28,31 as the primary outcome. In nine studies 5,28,29,31,32,33,34,35,38 univariate analyses showed a statistically significant positive association between maternal pre-pregnancy BMI and fat mass in offspring. This association remained significant after adjusting for confounders in six of the studies $28,29,31,32,34,38$. The relationship between maternal 
Methodological characteristic of articles assessing maternal pre-gestational BMI and/or gestational weight gain and offspring body composition in childhood.

\begin{tabular}{|c|c|c|c|c|c|}
\hline $\begin{array}{l}\text { Author (city, } \\
\text { country) }\end{array}$ & $\begin{array}{l}\text { Design/N/Score } \\
\text { Downs \& Black } 26\end{array}$ & Inclusion and exclusion criteria & $\begin{array}{l}\text { Maternal } \\
\text { exposure }\end{array}$ & $\begin{array}{l}\text { Indirect method } \\
\text { /Outcome age } \\
\text { measurement }\end{array}$ & Adjustment variables \\
\hline $\begin{array}{l}\text { Berkowitz et } \\
\text { al. } 34 \\
\text { (Philadelphia, } \\
\text { USA) }\end{array}$ & $\begin{array}{l}\text { Prospective cohort } \\
\qquad \begin{array}{c}N=78 \\
\text { Score }=14\end{array}\end{array}$ & $\begin{array}{l}\text { Inclusion: maternal age > } 18 \text { years } \\
\text { old, caucasian. Offspring healthy, } \\
\text { uncomplicated full-term births. } \\
\text { Exclusion: maternal gestational } \\
\text { diabetes. Offspring: gestational age } \\
<36 \text { or }>42 \text { weeks, low or high birth } \\
\text { weight for gestational age }\end{array}$ & $\begin{array}{l}\text { Maternal pre- } \\
\text { pregnancy } \\
\text { BMI }\end{array}$ & $\begin{array}{l}\text { DEXA, at } 6 \text { years } \\
\text { of age }\end{array}$ & $\begin{array}{l}\text { Maternal: family income, obesity risk. } \\
\text { Offspring: gender, weight at age two }\end{array}$ \\
\hline $\begin{array}{l}\text { Burdette et al. } 35 \\
\text { (Cincinnati, USA) }\end{array}$ & $\begin{array}{l}\text { Prospective cohort } \\
\qquad \begin{array}{l}\mathrm{N}=313 \\
\text { Score }=15\end{array}\end{array}$ & $\begin{array}{l}\text { Inclusion: father and mother of same } \\
\text { race. Offspring: full-term birth, no } \\
\text { chronic diseases }\end{array}$ & $\begin{array}{l}\text { Maternal pre- } \\
\text { pregnancy } \\
\text { BMI }\end{array}$ & $\begin{array}{l}\text { DEXA, at } 5 \text { years } \\
\text { of age }\end{array}$ & $\begin{array}{l}\text { Maternal: race, age, education, } \\
\text { smoking during pregnancy, marital } \\
\text { status, family income, nutrition } \\
\text { program. Offspring: gender, lean } \\
\text { mass, birth weight, breastfeeding, } \\
\text { overfeeding }\end{array}$ \\
\hline $\begin{array}{l}\text { Sewell et al. } 29 \\
\text { (Cleveland, USA) }\end{array}$ & $\begin{array}{l}\text { Prospective cohort } \\
\qquad N=220 \\
\text { Score }=15\end{array}$ & $\begin{array}{l}\text { Inclusion: pregnant women with normal } \\
\text { glucose tolerance, singleton pregnancy. } \\
\text { Exclusion: maternally or gestational } \\
\text { diabetes mellitus. Offspring: birth } \\
\text { weight }<2,000 \mathrm{~g} \text {, congenital anomalies }\end{array}$ & $\begin{array}{l}\text { Maternal pre- } \\
\text { pregnancy } \\
\text { BMI } \\
\text { Gestational } \\
\text { weight gain }\end{array}$ & $\begin{array}{l}\text { TOBEC, at } 72 \\
\text { hours after birth }\end{array}$ & $\begin{array}{c}\text { None } \\
\text { Maternal: glucose, smoking during } \\
\text { pregnancy. Offspring: gestational } \\
\text { age, gender }\end{array}$ \\
\hline $\begin{array}{l}\text { Gale et al. } 28 \\
\text { (Southampton, } \\
\text { UK) }\end{array}$ & $\begin{array}{l}\text { Prospective cohort } \\
\qquad \begin{array}{l}N=216 \\
\text { Score }=18\end{array}\end{array}$ & $\begin{array}{l}\text { Inclusion: maternal: caucasian, age } \geq 16 \\
\text { years, singleton pregnancy. } \\
\text { Exclusion: maternal gestational } \\
\text { diabetes, hormonal therapy for } \\
\text { pregnancy. Offspring: gestational age } \\
<17 \text { weeks }\end{array}$ & $\begin{array}{l}\text { Maternal pre- } \\
\text { pregnancy } \\
\text { BMI }\end{array}$ & $\begin{array}{l}\text { DEXA, at } 9 \text { years } \\
\text { of age }\end{array}$ & $\begin{array}{c}\text { Maternal: height, gestational weight } \\
\text { gain, smoking during pregnancy. } \\
\text { Offspring: height, birth weight, } \\
\text { breastfeeding, conditional } \\
\text { weight gain } \\
\text { Maternal: height, smoking during } \\
\text { pregnancy, pre-pregnancy BMI. } \\
\text { Offspring: height, birth weight, } \\
\text { breastfeeding, conditional } \\
\text { weight gain }\end{array}$ \\
\hline $\begin{array}{l}\text { Lawlor et al. } 38 \\
\text { (Avon, UK) }\end{array}$ & $\begin{array}{l}\text { Prospective cohort } \\
\qquad \begin{array}{l}N=3,263 \\
\text { Score }=17\end{array}\end{array}$ & Inclusion: singleton pregnancy & $\begin{array}{l}\text { Maternal pre- } \\
\text { pregnancy } \\
\text { BMI }\end{array}$ & $\begin{array}{l}\text { DEXA, at 9-11 } \\
\text { years of age }\end{array}$ & $\begin{array}{c}\text { Height and height squared at time } \\
\text { of DEXA assessment, gender, } \\
\text { age, family social class, parental } \\
\text { education, parity, parental smoking at } \\
\text { time of pregnancy, offspring pubertal } \\
\text { status at time of DEXA assessment, } \\
\text { breastfeeding }\end{array}$ \\
\hline $\begin{array}{l}\text { Hull et al. } 5 \\
\text { (Oklahoma City, } \\
\text { USA) }\end{array}$ & $\begin{array}{l}\text { Prospective cohort } \\
\qquad \begin{array}{c}\mathrm{N}=72 \\
\text { Score }=15\end{array}\end{array}$ & $\begin{array}{l}\text { Inclusion: maternal: age at delivery } \\
\text { 18-45 years old. Offspring: healthy, full- } \\
\text { term birth. } \\
\text { Exclusion: maternal smoking and } \\
\text { excessive alcohol consumption } \\
\text { during pregnancy, diabetes type 1, } \\
2 \text { or gestational diabetes. Offspring: } \\
\text { congenital anomalies }\end{array}$ & $\begin{array}{l}\text { Maternal pre- } \\
\text { pregnancy } \\
\text { BMl }\end{array}$ & $\begin{array}{l}\text { ADP, at first } 35 \\
\text { days of age }\end{array}$ & Age of children at time of visit \\
\hline
\end{tabular}

(continues) 
Table 1 (continued)

\begin{tabular}{|c|c|c|c|c|c|}
\hline $\begin{array}{l}\text { Author (city, } \\
\text { country) }\end{array}$ & $\begin{array}{l}\text { Design/N/Score } \\
\text { Downs \& Black } 26\end{array}$ & Inclusion and exclusion criteria & $\begin{array}{l}\text { Maternal } \\
\text { exposure }\end{array}$ & $\begin{array}{l}\text { Indirect method } \\
\text { /Outcome age } \\
\text { measurement }\end{array}$ & Adjustment variables \\
\hline $\begin{array}{l}\text { Crozier et al. } 42 \\
\text { (Southampton, } \\
\text { UK) }\end{array}$ & $\begin{array}{l}\text { Prospective cohort } \\
\qquad \begin{array}{l}\mathrm{N}=948 \\
\text { Score }=15\end{array}\end{array}$ & & $\begin{array}{l}\text { Gestational } \\
\text { weight gain }\end{array}$ & $\begin{array}{l}\text { ADP, at birth, age } \\
4 \text { years, age } 6 \\
\text { years of age }\end{array}$ & $\begin{array}{c}\text { Maternal: pre-pregnancy BMI, } \\
\text { smoking during pregnancy, age, } \\
\text { height, parity, education. Offspring: } \\
\text { birth weight, height, duration of } \\
\text { breastfeeding, gender, age at time } \\
\text { of assessment }\end{array}$ \\
\hline $\begin{array}{l}\text { Hull et al. } 6 \\
\text { (New York, USA) }\end{array}$ & $\begin{array}{l}\text { Prospective cohort } \\
\qquad \begin{array}{l}N=306 \\
\text { Score }=15\end{array}\end{array}$ & $\begin{array}{l}\text { Inclusion: healthy, full-term birth, } \\
\text { singleton pregnancy. } \\
\text { Exclusion: maternal diabetes, } \\
\text { hypertension or pre-eclampsia. } \\
\text { Offspring: congenital anomalies, } \\
\text { admission to ICU }\end{array}$ & $\begin{array}{l}\text { Maternal pre- } \\
\text { pregnancy } \\
\text { BMI } \\
\text { Gestational } \\
\text { weight gain }\end{array}$ & $\begin{array}{l}\text { ADP, at } 24-48 \\
\text { hours after birth }\end{array}$ & $\begin{array}{l}\text { Maternal: skin color/race, age. } \\
\text { Offspring: gender, gestational age, } \\
\text { age at the time of the assessment }\end{array}$ \\
\hline $\begin{array}{l}\text { Au et al. } 27 \\
\text { (Sydney, } \\
\text { Australia) }\end{array}$ & $\begin{array}{l}\text { Retrospective } \\
\text { cohort } \\
N=599 \\
\text { Score }=15\end{array}$ & $\begin{array}{l}\text { Inclusion: full-term birth, living within the } \\
\text { geographical area of the health service. } \\
\text { Exclusion: maternal gestational diabetes. } \\
\text { Offspring: congenital anomalies or } \\
\text { admission to ICU }\end{array}$ & $\begin{array}{l}\text { Maternal pre- } \\
\text { pregnancy } \\
\text { BMI }\end{array}$ & $\begin{array}{l}\text { ADP, at } 48 \text { hours } \\
\text { after birth }\end{array}$ & $\begin{array}{l}\text { Maternal: ethnicity, parity, } \\
\text { hypertension, gestational weight } \\
\text { gain, gestational diabetes mellitus } \\
\text { status. Offspring: gender, } \\
\text { gestational age }\end{array}$ \\
\hline $\begin{array}{l}\text { Josefson et al. } 41 \\
\text { (Chicago, USA) }\end{array}$ & $\begin{array}{l}\text { Prospective cohort } \\
\qquad \begin{array}{c}N=38 \\
\text { Score }=13\end{array}\end{array}$ & $\begin{array}{l}\text { Inclusion: maternal normal diabetes } \\
\text { screening test in normal-weight }\end{array}$ & $\begin{array}{l}\text { Gestational } \\
\text { weight gain }\end{array}$ & $\begin{array}{l}\text { ADP, at } 72 \text { hours } \\
\text { after birth }\end{array}$ & None \\
\hline $\begin{array}{l}\text { Henriksson et } \\
\text { al. } 44 \text { (Linkoping, } \\
\text { Sweden) }\end{array}$ & $\begin{array}{l}\text { Prospective cohort } \\
\qquad \begin{array}{l}\mathrm{N}=312 \\
\text { Score }=15\end{array}\end{array}$ & $\begin{array}{l}\text { Inclusion: healthy offspring, singleton, } \\
\text { full-term. } \\
\text { Exclusion: Maternal: pre-eclampsia, lack } \\
\text { of pregnancy weight, serious disease. }\end{array}$ & $\begin{array}{l}\text { Gestational } \\
\text { weight gain }\end{array}$ & $\begin{array}{l}\text { ADP, at } 2 \text { to } 11 \\
\text { days of age }\end{array}$ & $\begin{array}{l}\text { Maternal: smoking habits, parity, } \\
\text { educational level. Offspring: height }\end{array}$ \\
\hline $\begin{array}{l}\text { Carlsen et al. } 32 \\
\text { (Copenhagen, } \\
\text { Denmark) }\end{array}$ & $\begin{array}{l}\text { Randomized } \\
\text { controlled trial } \\
\qquad \begin{array}{c}\mathrm{N}=311 \\
\text { Score }=24\end{array}\end{array}$ & $\begin{array}{l}\text { Inclusion: offspring: singleton, full-term. } \\
\text { Exclusion: maternal; chronic disease, } \\
\text { pre-eclampsia. Offspring: congenital } \\
\text { malformations, admission to ICU }\end{array}$ & $\begin{array}{l}\text { Maternal pre- } \\
\text { pregnancy } \\
\text { BMI }\end{array}$ & $\begin{array}{l}\text { DEXA, within } 48 \\
\text { hours after at birth }\end{array}$ & $\begin{array}{c}\text { Maternal: age, education level, } \\
\text { smoking habits }\end{array}$ \\
\hline $\begin{array}{l}\text { Estampador } \\
\text { et al. } 43 \\
\text { (Vasterbotten, } \\
\text { Sweden) }\end{array}$ & $\begin{array}{l}\text { Prospective cohort } \\
\qquad \begin{array}{c}\mathrm{N}=33 \\
\text { Score }=23\end{array}\end{array}$ & Inclusion: singleton pregnancy & $\begin{array}{l}\text { Rate of } \\
\text { gestational } \\
\text { weight gain }\end{array}$ & $\begin{array}{l}\text { ADP, at } 4 \text { months } \\
\text { of age }\end{array}$ & None \\
\hline $\begin{array}{l}\text { Tanvig et al. } 39 \\
\text { (Odense, } \\
\text { Denmark) }\end{array}$ & $\begin{array}{l}\text { Randomized } \\
\text { controlled trial } \\
\qquad \begin{array}{c}\mathrm{N}=86 \\
\text { Score }=23\end{array}\end{array}$ & $\begin{array}{l}\text { Inclusion: offspring: singleton, healthy, } \\
\text { caucasian mother. } \\
\text { Exclusion: maternal diabetes, } \\
\text { pre-existing hypertensive disorders, } \\
\text { mental illness }\end{array}$ & $\begin{array}{l}\text { Maternal pre- } \\
\text { pregnancy } \\
\text { BMI }\end{array}$ & $\begin{array}{c}\text { DEXA, at } 2.5 \text { to } 3 \\
\text { years of age }\end{array}$ & None \\
\hline $\begin{array}{l}\text { Perng et al. } 30 \\
\text { (Boston, USA) }\end{array}$ & $\begin{array}{l}\text { Prospective cohort } \\
\qquad \begin{array}{l}\mathrm{N}=848 \\
\text { Score }=16\end{array}\end{array}$ & $\begin{array}{l}\text { Inclusion: singleton pregnancy. } \\
\text { Exclusion: maternal diabetes, gestation } \\
\text { length less than } 34 \text { weeks }\end{array}$ & $\begin{array}{l}\text { Maternal pre- } \\
\text { pregnancy } \\
\text { BMI } \\
\text { Gestational } \\
\text { weight gain }\end{array}$ & $\begin{array}{l}\text { DEXA, at 6-10 } \\
\text { years of age }\end{array}$ & Maternal race/ethnicity, parity \\
\hline $\begin{array}{l}\text { Pereira-da-Silva } \\
\text { et al. } 40 \text { (Lisbon, } \\
\text { Portugal) }\end{array}$ & $\begin{array}{l}\text { Retrospective } \\
\text { cohort } \\
N=100 \\
\text { Score }=15\end{array}$ & $\begin{array}{l}\text { Inclusion: proper medical surveillance, } \\
\text { singleton full-term neonate, adequade } \\
\text { gestational age *, healthy offspring } \\
\text { Exclusion: adverse conditions potentially } \\
\text { affecting fetal nutrition and growth, } \\
\text { reported consumption of alcohol, } \\
\text { tobacco, or illicit drugs }\end{array}$ & $\begin{array}{l}\text { Maternal pre- } \\
\text { pregnancy } \\
\text { BMI }\end{array}$ & $\begin{array}{l}\text { ADP, at } 72 \text { hours } \\
\text { after birth }\end{array}$ & $\begin{array}{l}\text { Percentage of total energy value } \\
\text { from carbohydrate or from protein }\end{array}$ \\
\hline
\end{tabular}

(continues) 
Table 1 (continued)

\begin{tabular}{|c|c|c|c|c|c|}
\hline $\begin{array}{l}\text { Author (city, } \\
\text { country) }\end{array}$ & $\begin{array}{l}\text { Design/N/Score } \\
\text { Downs \& Black } 26\end{array}$ & Inclusion and exclusion criteria & $\begin{array}{l}\text { Maternal } \\
\text { exposure }\end{array}$ & $\begin{array}{l}\text { Indirect method } \\
\text { /Outcome age } \\
\text { measurement }\end{array}$ & Adjustment variables \\
\hline $\begin{array}{l}\text { Shapiro et al. } 33 \\
\text { (Denver, USA) }\end{array}$ & $\begin{array}{l}\text { Prospective cohort } \\
\qquad \begin{array}{c}\mathrm{N}=951 \\
\text { Score }=16\end{array}\end{array}$ & $\begin{array}{l}\text { Exclusion: preexisting diabetes type } 1 \text { or } \\
\text { 2, a prior premature birth or fetal death, } \\
\text { asthma with steroid management, } \\
\text { serious psychiatric illness or a current } \\
\text { multiple pregnancy }\end{array}$ & $\begin{array}{l}\text { Maternal pre- } \\
\text { pregnancy } \\
\text { BMI }\end{array}$ & $\begin{array}{l}\text { ADP, at } 48 \text { hours } \\
\text { after birth }\end{array}$ & \\
\hline $\begin{array}{l}\text { Starling et al. } 37 \\
\text { (Denver, USA) }\end{array}$ & $\begin{array}{l}\text { Prospective cohort } \\
\qquad \begin{array}{l}\mathrm{N}=826 \\
\text { Score }=16\end{array}\end{array}$ & $\begin{array}{l}\text { Exclusion: preexisting diabetes type } 1 \text { or } \\
\text { 2, a prior premature birth or fetal death, } \\
\text { asthma with steroid management, } \\
\text { serious psychiatric illness or a current } \\
\text { multiple pregnancy }\end{array}$ & $\begin{array}{l}\text { Maternal pre- } \\
\text { pregnancy- } \\
\text { BMI }\end{array}$ & $\begin{array}{c}\text { ADP, at } \leq 3 \text { days } \\
\text { of age }\end{array}$ & \\
\hline $\begin{array}{l}\text { Castillo et al. } 31 \\
\text { (Pelotas, Brazil) }\end{array}$ & $\begin{array}{l}\text { Prospective cohort } \\
\qquad \begin{array}{l}\mathrm{N}=3,156 \\
\text { Score }=18\end{array}\end{array}$ & Inclusion: singleton pregnancy & $\begin{array}{l}\text { Maternal pre- } \\
\text { pregnancy } \\
\text { BMI } \\
\text { Gestational } \\
\text { weight gain }\end{array}$ & ADP, at 6 years & $\begin{array}{l}\text { Maternal: family income, schooling } \\
\text { at birth, skin color, age, parity, } \\
\text { smoking and alcohol consumption } \\
\text { during pregnancy, history of arterial } \\
\text { hypertension, history of diabetes } \\
\text { mellitus, gestational age. } \\
\text { Offspring: birthweight and sex. }\end{array}$ \\
\hline $\begin{array}{l}\text { Andrés et al. } 36 \\
\text { (Kansas City, } \\
\text { USA) }\end{array}$ & $\begin{array}{l}\text { Prospective cohort } \\
\qquad \begin{array}{c}\mathrm{N}=325 \\
\text { Score }=17\end{array}\end{array}$ & $\begin{array}{l}\text { Inclusion: pregnancy uncomplicated, } \\
\text { nonsmokers, who denied alcohol use } \\
\text { during pregnancy. Singleton full-term } \\
\text { offspring, exclusively breast-fed until at } \\
\text { least age } 6 \text { months }\end{array}$ & $\begin{array}{l}\text { Maternal pre- } \\
\text { pregnancy } \\
\text { BMI }\end{array}$ & DEXA, at 6 years & $\begin{array}{l}\text { Maternal: race, gestational age. } \\
\text { Offspring: birthweight and mode of } \\
\text { infant feeding. }\end{array}$ \\
\hline
\end{tabular}

ADP: plethysmography; DEXA: dual-energy X-ray absorptiometry; ICU: intensive care unit; TOBEC: total body electrical conductivity.

* Birth weight $>3$ rd and $97^{\text {th }}$ percentiles for gestational age and gender).

Note: rate of gestational weight gain in mid-pregnancy or in late-pregnancy (kg per week).

pre-pregnancy BMI and body fat mass in children was assessed in 13 studies 5,6,27,29,31,32,33,34,35, $36,37,39,40$ and a positive association was reported only following univariate analyses in six studies $5,29,31,32,33,34$.

Eight studies included in the meta-analysis $5,6,27,29,31,32,33,39$ assessed body fat percent and fat mass in infants in the first months of life to six years of age. The SMD between infants of normal weight women and those of overweight or obese women before pregnancy were relatively small: 0.31 (95\%CI: 0.23 ; 0.39 ) and 0.38 (95\%CI: 0.30 ; $0.46)$ for body fat percent and fat mass, respectively $\left(\mathrm{I}^{2}=28 \%\right.$ and $18.5 \%$; and $\mathrm{p}$ value for test of heterogeneity of 0.21 and 0.29 , respectively) (Figure 2).

Begg's funnel plots and Egger's publication bias plots were used to assess the potential publication bias for body fat percent and fat mass. No publication bias was detected by Egger's test ( $\mathrm{p}$-value $=0.49$ for body fat percent and $\mathrm{p}$-value $=$ 0.60 for fat mass).

\section{- Maternal pre-pregnancy BMI and offspring lean mass or fat-free mass}

The relationship of maternal pre-pregnancy BMI with lean mass 34,38 , fat-free mass 5,6,28,29,31,32,33,39, fat-free mass percent ${ }^{36}$ and LMI 28,31 was assessed in 11 studies (Table 2). Four studies reported a statistically significant positive association following a univariate analysis 28,31,34,38 and two studies 31,36 after adjusted analysis. In contrast, Gale et al. 28 reported a significant positive association between pre-pregnancy BMI and LMI in only male offspring and this was no longer significant following adjusted analysis. Furthermore, Hull et al. 5 reported an inverse association. In the aggregate measure analysis, SMD in fat-free mass between children of normal weight women and those of overweight or obese women was significant: 0.21 (95\%CI: $0.02 ; 0.40), \mathrm{I}^{2}=84.9 \%$, test for heterogeneity $\mathrm{p}$-value $=0.000$ (Figure 2 ). No publication bias was detected by Egger's test, $\mathrm{p}$-value = 0.12 for fat-free mass.

In relation to SMD in fat-free mass aggregated analyses, heterogeneity was detected in the visu- 
Table 2

Maternal pre-pregnacy body mass index (BMI) and offspring body composition in childhood.

\begin{tabular}{|c|c|c|c|c|}
\hline Author & $\begin{array}{l}\text { Maternal exposure } \\
\text { (pre-gestational BMI) }\end{array}$ & Offspring outcome/Measures of effect & Crude analyses & Adjusted analyses \\
\hline \multirow{2}{*}{\multicolumn{5}{|c|}{$\begin{array}{l}\text { Studies that assessed } \\
\text { body fat percent }\end{array}$}} \\
\hline & & & & \\
\hline \multirow[t]{4}{*}{ Berkowitz et al. 34} & High risk & $\begin{array}{l}\text { Body fat \% (percentage point) at } 4 \text { years, } \\
\text { mean (SD) }\end{array}$ & $21.0(6.2)$ & - \\
\hline & Low risk & & $20.3(3.4)$ & - \\
\hline & High risk & $\begin{array}{l}\text { Body fat \% (percentage point) at } 6 \text { years, } \\
\text { mean (SD) } / \beta \text { (SE) }\end{array}$ & $24.7(11.8)$ & $112.3(77.4)$ \\
\hline & Low risk & & $18.8(4.5)$ * & Reference \\
\hline \multirow[t]{2}{*}{ Sewell et al. 29} & $<25 \mathrm{~kg} / \mathrm{m}^{2}$ & $\begin{array}{l}\text { Body fat \% (percentage point) at } 72 \text { hours, } \\
\text { mean (SD) }\end{array}$ & $9.6(4.3)$ & - \\
\hline & $\geq 25 \mathrm{~kg} / \mathrm{m}^{2}$ & & $11.0(4.7)$ * & - \\
\hline \multirow[t]{2}{*}{ Burdette et al. 35} & $\geq 30 \mathrm{~kg} / \mathrm{m}^{2}$ & Hight adiposity (\%) at 6 years, \%/OR $(95 \% \mathrm{Cl})$ & 30.8 & $0.95(0.4 ; 2.1)$ \\
\hline & $<30 \mathrm{~kg} / \mathrm{m}^{2}$ & & 23.4 & Reference \\
\hline \multirow[t]{2}{*}{ Hull et al. 5} & $<25 \mathrm{~kg} / \mathrm{m}^{2}$ & $\begin{array}{l}\text { Body fat \% (percentage point) at } 35 \text { days, } \\
\text { mean (SD) }\end{array}$ & $12.5(4.2)$ & - \\
\hline & $\geq 25 \mathrm{~kg} / \mathrm{m}^{2}$ & & $13.6(4.3)$ * & - \\
\hline \multirow[t]{3}{*}{ Hull et al. 6} & $<25 \mathrm{~kg} / \mathrm{m}^{2}$ & $\begin{array}{l}\text { Body fat \% (percentage point) at 24-48 hours, } \\
\text { mean (SD) }\end{array}$ & $11.7(4.1)$ & - \\
\hline & $25-30 \mathrm{~kg} / \mathrm{m}^{2}$ & & $13.0(4.7)$ & - \\
\hline & $>30 \mathrm{~kg} / \mathrm{m}^{2}$ & & $14.6(4.3)$ & - \\
\hline \multirow[t]{5}{*}{ Au et al. 27} & $<18.5 \mathrm{~kg} / \mathrm{m}^{2}$ & $\begin{array}{l}\text { Body fat } \% \text { (percentage point) at } 48 \text { hours, mean } \\
\qquad(\mathrm{SD}) / \beta(95 \% \mathrm{Cl})\end{array}$ & $8.8(4.1)$ & $-0.2(-1.5 ; 1.2)$ \\
\hline & $18-24 \mathrm{~kg} / \mathrm{m}^{2}$ & & $9.0(4.2)$ & Reference \\
\hline & $25-29 \mathrm{~kg} / \mathrm{m}^{2}$ & & $9.9(4.8)$ & $1.2(0.3 ; 2.1)$ * \\
\hline & $30-34 \mathrm{~kg} / \mathrm{m}^{2}$ & & $9.0(4.5)$ & $-0.9(-2.8 ; 0.9)$ \\
\hline & $>35 \mathrm{~kg} / \mathrm{m}^{2}$ & & - & $3.1(0.7 ; 5.5)$ * \\
\hline \multirow[t]{2}{*}{ Carlsen at al. 32} & $18-24 \mathrm{~kg} / \mathrm{m}^{2}$ & $\begin{array}{l}\text { Body fat \% (percentage point) at } 48 \text { hours, mean } \\
\qquad(\mathrm{SD}) / \beta(95 \% \mathrm{Cl})\end{array}$ & $8.8(3.6)$ * & Reference \\
\hline & $\geq 30 \mathrm{~kg} / \mathrm{m}^{2}$ & & $11.2(4.3)$ & $3.1(2.0 ; 4.1)$ \\
\hline \multirow[t]{2}{*}{ Tanvig et al. 39} & $18-24 \mathrm{~kg} / \mathrm{m}^{2}$ & $\begin{array}{l}\text { Body fat \% (percentage point) at } 2.5-3 \text { years, } \\
\text { mean }(95 \% \mathrm{Cl})\end{array}$ & $21.3(19.5 ; 23.1)$ & - \\
\hline & $\geq 30 \mathrm{~kg} / \mathrm{m}^{2}$ & & $21.6(19.4 ; 23.8)$ & - \\
\hline \multirow{2}{*}{$\begin{array}{l}\text { Pereira-da-Silva et } \\
\text { al. } 40\end{array}$} & $\mathrm{~kg} / \mathrm{m}^{2}$ & Body fat $\%$ (percentage point) at 72 hours, $\beta$ & 0.2 & - \\
\hline & $\geq 25 \mathrm{~kg} / \mathrm{m}^{2}$ & Bady fat $\%$ (percentage point) at 72 hours, $\beta$ & 0.9 & - \\
\hline \multirow[t]{4}{*}{ Shapiro et al. 33} & $<25 \mathrm{~kg} / \mathrm{m}^{2}$ & $\begin{array}{l}\text { Body fat \% (percentage point) at } 48 \text { hours, } \\
\text { mean (SD) }\end{array}$ & $8.8(3.8)$ & - \\
\hline & $25-29 \mathrm{~kg} / \mathrm{m}^{2}$ & & $9.4(3.9)$ & - \\
\hline & $\geq 30 \mathrm{~kg} / \mathrm{m}^{2}$ & & $10.1(4.0)$ & - \\
\hline & $\mathrm{kg} / \mathrm{m}^{2}$ & $\begin{array}{l}\text { Body fat } \% \text { (percentage point) at } 48 \text { hours, } \beta \\
\qquad(95 \% \mathrm{Cl})\end{array}$ & $0.2(0.1 ; 0.2)$ & $0.1(0.04 ; 0.2)$ \\
\hline Starling et al. 37 & $\mathrm{~kg} / \mathrm{m}^{2}$ & $\begin{array}{l}\text { Body fat } \% \text { (percentage point) at } 3 \text { days, } \beta \\
\qquad(95 \% \mathrm{Cl})\end{array}$ & - & $0.1(0.1 ; 0.2)$ \\
\hline \multirow[t]{5}{*}{ Castillo et al. 31} & $\mathrm{~kg} / \mathrm{m}^{2}$ & $\begin{array}{l}\text { Body fat } \% \text { (percentage point) at } 6 \text { years, } \beta \\
\qquad(95 \% \mathrm{Cl})\end{array}$ & $0.3(0.3 ; 0.4)$ & $0.18(0.03 ; 0.3)$ \\
\hline & $<18.5 \mathrm{~kg} / \mathrm{m}^{2}$ & $\begin{array}{l}\text { Body fat \% (percentage point) at } 6 \text { years, } \\
\text { mean (SD) }\end{array}$ & $20.1(5.6)^{\star \star}$ & \\
\hline & $18.5-24 \mathrm{~kg} / \mathrm{m}^{2}$ & & $23.0(7.5)$ & \\
\hline & $25-29 \mathrm{~kg} / \mathrm{m}^{2}$ & & $24.7(8.4)$ & \\
\hline & $\geq 30 \mathrm{~kg} / \mathrm{m}^{2}$ & & $26.8(9.5)$ & \\
\hline
\end{tabular}

(continues) 
Table 2 (continued)

\begin{tabular}{|c|c|c|c|c|}
\hline Author & $\begin{array}{l}\text { Maternal exposure } \\
\text { (pre-gestational BMI) }\end{array}$ & Offspring outcome/Measures of effect & Crude analyses & Adjusted analyses \\
\hline \multicolumn{5}{|l|}{$\begin{array}{l}\text { Studies that assessed } \\
\text { fat mass }\end{array}$} \\
\hline \multirow[t]{4}{*}{ Berkowitz et al. 34} & High risk & Fat mass $(\mathrm{kg})$ at age 4 years, mean (SD) & $3.7(1.6)$ & - \\
\hline & Low risk & & $3.2(0.8)$ & - \\
\hline & High risk & Fat mass $(\mathrm{kg})$ at 6 years, mean $(\mathrm{SD}) / \beta(\mathrm{SE})$ & $6.7(5.7)$ & $0.3(0.1)^{*}$ \\
\hline & Low risk & & $3.8(1.2) *$ & Reference \\
\hline \multirow[t]{2}{*}{ Sewell et al. 29} & $<25 \mathrm{~kg} / \mathrm{m}^{2}$ & Fat mass (g) at 72 hours, mean (SD) & $331(179)$ & - \\
\hline & $\geq 25 \mathrm{~kg} / \mathrm{m}^{2}$ & & $406(221)$ * & - \\
\hline \multirow[t]{2}{*}{ Burdette et al. 35} & $\geq 30 \mathrm{~kg} / \mathrm{m}^{2}$ & Fat mass $(\mathrm{kg})$ at 5 years, mean $(\mathrm{SD}) / \beta(95 \% \mathrm{Cl})$ & $5.0(0.2)$ & $0.5(0.02 ; 0.9)$ \\
\hline & $<30 \mathrm{~kg} / \mathrm{m}^{2}$ & & $4.5(0.1) *$ & Reference \\
\hline \multirow[t]{2}{*}{ Gale et al. 28} & $\mathrm{~kg} / \mathrm{m}^{2}$ & FMI (SDS) at 9 years, male, $\beta(95 \% \mathrm{Cl})$ & $0.2(-0.04 ; 0.4)$ & $0.3(0.04 ; 0.5)$ \\
\hline & & FMI (SDS) at 9 years, female $\beta(95 \% \mathrm{Cl})$ & $0.4(0.3 ; 0.5)$ & $0.4(0.3 ; 0.6)$ \\
\hline Lawlor et al. 38 & SD & $\begin{array}{c}\text { Fat mass (z-score) at 9-11 years, } \\
\text { mean *** }(95 \% \mathrm{Cl})\end{array}$ & $0.3(0.2 ; 0.3)$ & $0.3(0.2 ; 0.3)$ \\
\hline \multirow[t]{4}{*}{ Hull et al. 5} & $<25 \mathrm{~kg} / \mathrm{m}^{2}$ & Fat mass (g) at 35 days, mean (SD) & $414.1(264.2)$ & - \\
\hline & $\geq 25 \mathrm{~kg} / \mathrm{m}^{2}$ & & $448.3(262.2) *$ & - \\
\hline & $25-30 \mathrm{~kg} / \mathrm{m}^{2}$ & & $450.9(216.3)$ & - \\
\hline & $>30 \mathrm{~kg} / \mathrm{m}^{2}$ & & $489.6(158.7)$ & - \\
\hline \multirow[t]{2}{*}{ Carlsen at al. 32} & $18-24 \mathrm{~kg} / \mathrm{m}^{2}$ & Fat mass $(\mathrm{g})$ at 48 hours, mean $(\mathrm{SD}) / \beta(95 \% \mathrm{Cl})$ & $331(163)$ * & Reference \\
\hline & $\geq 30 \mathrm{~kg} / \mathrm{m}^{2}$ & & $443(212)$ & $135(85 ; 186)$ \\
\hline \multirow{2}{*}{$\begin{array}{l}\text { Pereira-da-Silva et } \\
\text { al. } 40\end{array}$} & $\mathrm{~kg} / \mathrm{m}^{2}$ & Fat mass $(\mathrm{kg})$, at 72 hours, $\beta$ & 0.1 & - \\
\hline & $\geq 25 \mathrm{~kg} / \mathrm{m}^{2}$ & Fat mass $(\mathrm{kg})$, at 72 hours, $\beta(95 \% \mathrm{Cl})$ & 0.5 & $0.1(0.03 ; 0.2)^{*, \star \star \star}$ \\
\hline \multirow[t]{2}{*}{ Tanvig et al. 39} & $18-24 \mathrm{~kg} / \mathrm{m}^{2}$ & Fat mass $(\mathrm{kg})$ at $2.5-3$ years, mean $(95 \% \mathrm{Cl})$ & $2.3(2.1 ; 2.5)$ & - \\
\hline & $\geq 30 \mathrm{~kg} / \mathrm{m}^{2}$ & & $2.4(2.2 ; 2.7)$ & - \\
\hline Perng et al. 30 & per $5 \mathrm{~kg} / \mathrm{m}^{2}$ & Fat mass $(\mathrm{kg})$ at $6-10$ years, $\beta(95 \% \mathrm{Cl})$ & & $0.9(0.7 ; 1.1)$ \\
\hline \multirow[t]{3}{*}{ Shapiro et al. 33} & $<25 \mathrm{~kg} / \mathrm{m}^{2}$ & Fat mass (g) at 48 hours, mean (SD) & $280.5(143.2)$ & - \\
\hline & $25-29 \mathrm{~kg} / \mathrm{m}^{2}$ & & $306.7(151.9)$ & - \\
\hline & $\geq 30 \mathrm{~kg} / \mathrm{m}^{2}$ & & $332.6(159.6)$ & - \\
\hline Starling et al. 37 & $\mathrm{~kg} / \mathrm{m}^{2}$ & Fat mass $(\mathrm{g})$ at 3 days, $\beta(95 \% \mathrm{Cl})$ & & $5.2(3.5 ; 6.9)$ \\
\hline \multirow[t]{10}{*}{ Castillo et al. 31} & $\mathrm{~kg} / \mathrm{m}^{2}$ & Fat mass $(\mathrm{kg})$ at 6 years, $\beta(95 \% \mathrm{Cl})$ & $0.02(0.1 ; 0.2)$ & $0.1(0.03 ; 0.2)$ \\
\hline & $\mathrm{kg} / \mathrm{m}^{2}$ & $\mathrm{FMI}\left(\mathrm{kg} / \mathrm{m}^{2}\right)$ at 6 years, $\beta(95 \% \mathrm{Cl})$ & $0.1(0.1 ; 0.1)$ & $0.07(0.02 ; 0.1)$ \\
\hline & $<18.5 \mathrm{~kg} / \mathrm{m}^{2}$ & Fat mass $(\mathrm{kg})$ at 6 years, mean (SD) & $4.5(1.9) * \star$ & \\
\hline & $18.5-24 \mathrm{~kg} / \mathrm{m}^{2}$ & & $5.9(3.2)$ & \\
\hline & $25-29 \mathrm{~kg} / \mathrm{m}^{2}$ & & $6.9(4.0)$ & \\
\hline & $\geq 30 \mathrm{~kg} / \mathrm{m}^{2}$ & $\mathrm{FMI}\left(\mathrm{kg} / \mathrm{m}^{2}\right)$ at 6 years, mean (SD) & $8.0(5.2)$ & \\
\hline & $<18.5 \mathrm{~kg} / \mathrm{m}^{2}$ & & $3.1(1.2) * *$ & \\
\hline & $18.5-24 \mathrm{~kg} / \mathrm{m}^{2}$ & & $4.0(2.0)$ & \\
\hline & $25-29 \mathrm{~kg} / \mathrm{m}^{2}$ & & $4.5(2.4)$ & \\
\hline & $\geq 30 \mathrm{~kg} / \mathrm{m}^{2}$ & & $5.3(3.0)$ & \\
\hline
\end{tabular}

(continues)

al inspection of the forest plot of studies included in the review, a finding that was supported by statistical evidence. Seeking for causes in the studies' descriptions, although the eligibility criteria in the seven studies were similar, there was heterogeneity due to variation in the study sample
$(<100, \geq 100)$ and in outcome age measurement ( $<1$ week and $\geq 1$ week). Methodological heterogeneity was detected at study design (prospective cohort and randomized controlled trial), indirect method to outcome measurement (TOBEC, ADP and DEXA) and at confounding control (crude 
Table 2 (continued)

\begin{tabular}{|c|c|c|c|c|}
\hline Author & $\begin{array}{l}\text { Maternal exposure } \\
\text { (pre-gestational BMI) }\end{array}$ & Offspring outcome/Measures of effect & Crude analyses & Adjusted analyses \\
\hline \multirow{3}{*}{\multicolumn{5}{|c|}{$\begin{array}{l}\text { Studies that assessed } \\
\text { lean mass or fat-free } \\
\text { mass }\end{array}$}} \\
\hline & & & & \\
\hline & & & & \\
\hline \multirow[t]{4}{*}{ Berkowitz et al. 34} & High risk & Lean mass $(\mathrm{kg})$ at 4 years, mean (SD) & $13.1(1.5)$ & - \\
\hline & Low risk & & $12.2(0.9)$ * & - \\
\hline & High risk & Lean mass $(\mathrm{kg})$ at 6 years, mean (SD) & $16.7(1.9)$ & - \\
\hline & Low risk & & $15.6(1.3)$ * & - \\
\hline \multicolumn{5}{|l|}{ Sewell et al. 29} \\
\hline & $<25 \mathrm{~kg} / \mathrm{m}^{2}$ & Fat-free mass (g) at 72 hours, mean (SD) & $2951(406)$ & - \\
\hline & $\geq 25 \mathrm{~kg} / \mathrm{m}^{2}$ & & $3023(410)$ & - \\
\hline \multirow[t]{2}{*}{ Gale et al. 28} & $\mathrm{~kg} / \mathrm{m}^{2}$ & LMI (SDS) at 9 years, male, $\beta(95 \% \mathrm{Cl})$ & $0.2(0.04 ; 0.4)$ & $0.2(-0.02 ; 0.4)$ \\
\hline & & LMI (SDS), at 9 years, female, $\beta(95 \% \mathrm{Cl})$ & $0.2(-0.1 ; 0.3)$ & $0.1(-0.1 ; 0.3)$ \\
\hline Lawlor et al. 38 & SD & $\begin{array}{c}\text { Lean mass (z-score) at 9-11 years, } \\
\text { mean }{ }^{\star \star \star}(95 \% \mathrm{Cl})\end{array}$ & $0.1(0.1 ; 0.1)$ & $0.1(0.1 ; 0.1)$ \\
\hline \multirow[t]{2}{*}{ Hull et al. 5} & $<25 \mathrm{~kg} / \mathrm{m}^{2}$ & Fat-free mass (g) at 35 days, mean (SD) & $3,310.5(344.6)$ & - \\
\hline & $\geq 25 \mathrm{~kg} / \mathrm{m}^{2}$ & & $3,162.2(313.4)$ * & - \\
\hline \multirow[t]{3}{*}{ Hull et al. 6} & $<25 \mathrm{~kg} / \mathrm{m}^{2}$ & Fat-free mass (g) at 24-48 hours, mean (SD) & $2,826.5(330.4)$ & - \\
\hline & $25-30 \mathrm{~kg} / \mathrm{m}^{2}$ & & $2,903.3(407.4)$ & - \\
\hline & $>30 \mathrm{~kg} / \mathrm{m}^{2}$ & & $2,833.6(332.1)$ & - \\
\hline \multirow[t]{2}{*}{ Carlsen et al. 32} & $18-24 \mathrm{~kg} / \mathrm{m}^{2}$ & $\begin{array}{l}\text { Fat-free mass }(\mathrm{g}) \text { at } 48 \text { hours, mean }(\mathrm{SD}) / \beta \\
\qquad(95 \% \mathrm{Cl})\end{array}$ & $3,322(371)$ & Reference \\
\hline & $\geq 30 \mathrm{~kg} / \mathrm{m}^{2}$ & & $3,332(487)$ & $28(-69 ; 125)$ \\
\hline \multirow[t]{2}{*}{ Tanvig et al. 39} & $18-24 \mathrm{~kg} / \mathrm{m}^{2}$ & Fat-free mass $(\mathrm{kg})$ at $2.5-3$ years, mean $(95 \% \mathrm{Cl})$ & $10.9(10.6 ; 11.2)$ & - \\
\hline & $\geq 30 \mathrm{~kg} / \mathrm{m}^{2}$ & & $11.2(10.8 ; 11.7)$ & - \\
\hline \multirow{2}{*}{$\begin{array}{l}\text { Pereira-da-Silva et } \\
\text { al. } 40\end{array}$} & $\mathrm{~kg} / \mathrm{m}^{2}$ & Fat-free mass $(\mathrm{kg})$, at 72 hours, $\beta$ & 0.01 & - \\
\hline & $\geq 25 \mathrm{~kg} / \mathrm{m}^{2}$ & Fat-free mass $(\mathrm{kg})$, at 72 hours, $\beta(95 \% \mathrm{Cl})$ & 0.01 & $0.3(0.1 ; 0.4) *, * \star *$ \\
\hline \multirow[t]{3}{*}{ Shapiro et al. 33} & $<25 \mathrm{~kg} / \mathrm{m}^{2}$ & Fat-free mass (g) at 48 hours, mean (SD) & $2,830.7(324.8)$ & - \\
\hline & $25-29 \mathrm{~kg} / \mathrm{m}^{2}$ & & $2,877.0(366.7)$ & - \\
\hline & $\geq 30 \mathrm{~kg} / \mathrm{m}^{2}$ & & $2,853.3(33.8)$ & - \\
\hline Starling et al. 37 & $\mathrm{~kg} / \mathrm{m}^{2}$ & Fat-free mass (g) at 3 days, $\beta(95 \% \mathrm{Cl})$ & & $7.7(4.5 ; 10.9)$ \\
\hline \multirow[t]{10}{*}{ Castillo et al. 31} & $\mathrm{~kg} / \mathrm{m}^{2}$ & Fat-free mass $(\mathrm{kg})$ at 6 years, $\beta(95 \% \mathrm{Cl})$ & $0.1(0.1 ; 0.14)$ & $0.13(0.11 ; 0.2)$ \\
\hline & $\mathrm{kg} / \mathrm{m}^{2}$ & FFMI $\left(\mathrm{kg} / \mathrm{m}^{2}\right)$ at 6 years, $\beta(95 \% \mathrm{Cl})$ & $0.05(0.04 ; 0.1)$ & $0.06(0.05 ; 0.1)$ \\
\hline & $<18.5 \mathrm{~kg} / \mathrm{m}^{2}$ & Fat-free mass $(\mathrm{kg})$ at 6 years, mean (SD) & $17.3(2.3) \star \star$ & \\
\hline & $18.5-24 \mathrm{~kg} / \mathrm{m}^{2}$ & & $18.5(2.7)$ & \\
\hline & $25-29 \mathrm{~kg} / \mathrm{m}^{2}$ & & $19.3(2.9)$ & \\
\hline & $\geq 30 \mathrm{~kg} / \mathrm{m}^{2}$ & & $19.6(3.3)$ & \\
\hline & $<18.5 \mathrm{~kg} / \mathrm{m}^{2}$ & FFMI $\left(\mathrm{kg} / \mathrm{m}^{2}\right)$ at 6 years, mean (SD) & $12.1(1.0)^{\star \star}$ & \\
\hline & $18.5-24 \mathrm{~kg} / \mathrm{m}^{2}$ & & $12.6(1.1)$ & \\
\hline & $25-29 \mathrm{~kg} / \mathrm{m}^{2}$ & & $12.8(1.1)$ & \\
\hline & $\geq 30 \mathrm{~kg} / \mathrm{m}^{2}$ & & $13.2(1.3)$ & \\
\hline
\end{tabular}

95\%Cl: 95\% confidence interval; $\beta$ : beta coefficient; BMI: body mass index; FFMI: fat free mass index; FMI: fat mass index (kg/m²); LMI: lean mass index $(\mathrm{kg} / \mathrm{m} 2)$; SD: standard deviation; SE: standard error; SDS: standard deviation score; OR: odds ratio.

Note: high risk group: pre-gestational BMI greater than the 66th percentile or lower than the 33rd percentile;

* $p$-value: statistically significant;

** Mean difference;

*** In males. 
Figure 2

Standardized mean differences in infant body composition by mother pre-pregnancy body mass index (BMI) group, 2015.

2a) Body fat percent/Maternal pre-pregnancy BMI

$$
<25.0 \mathrm{~kg} / \mathrm{m}^{2} \quad \geq 25.0 \mathrm{~kg} / \mathrm{m}^{2}
$$

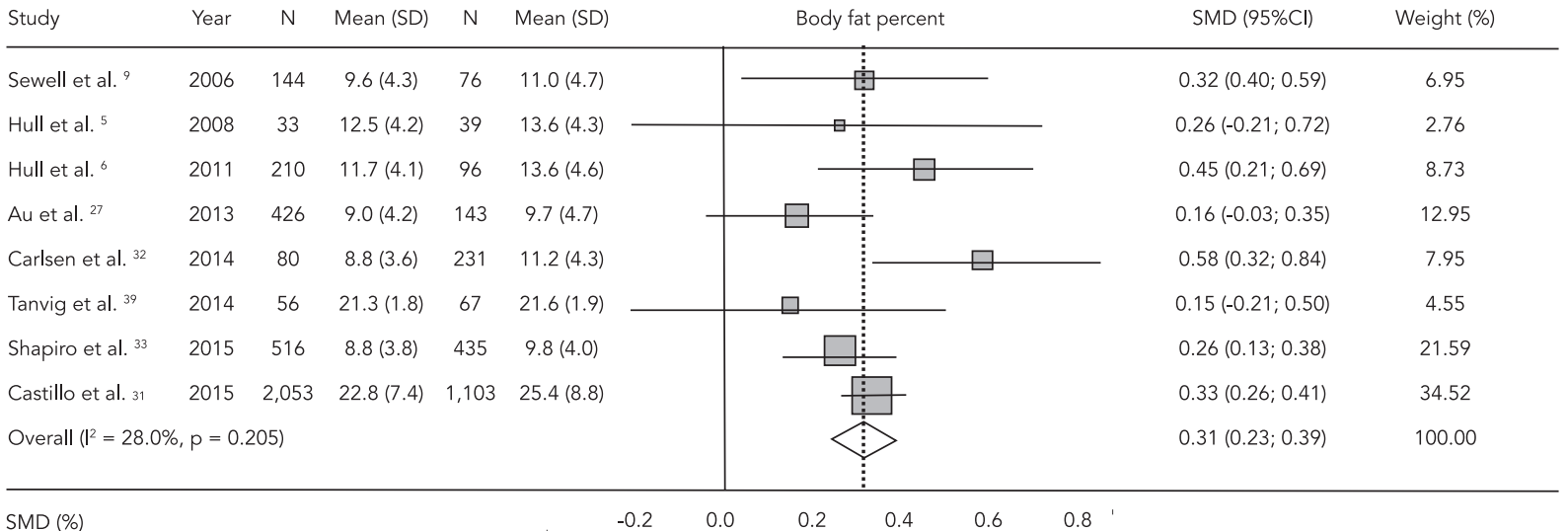

2b) Fat mass/Maternal pre-pregnancy BMI

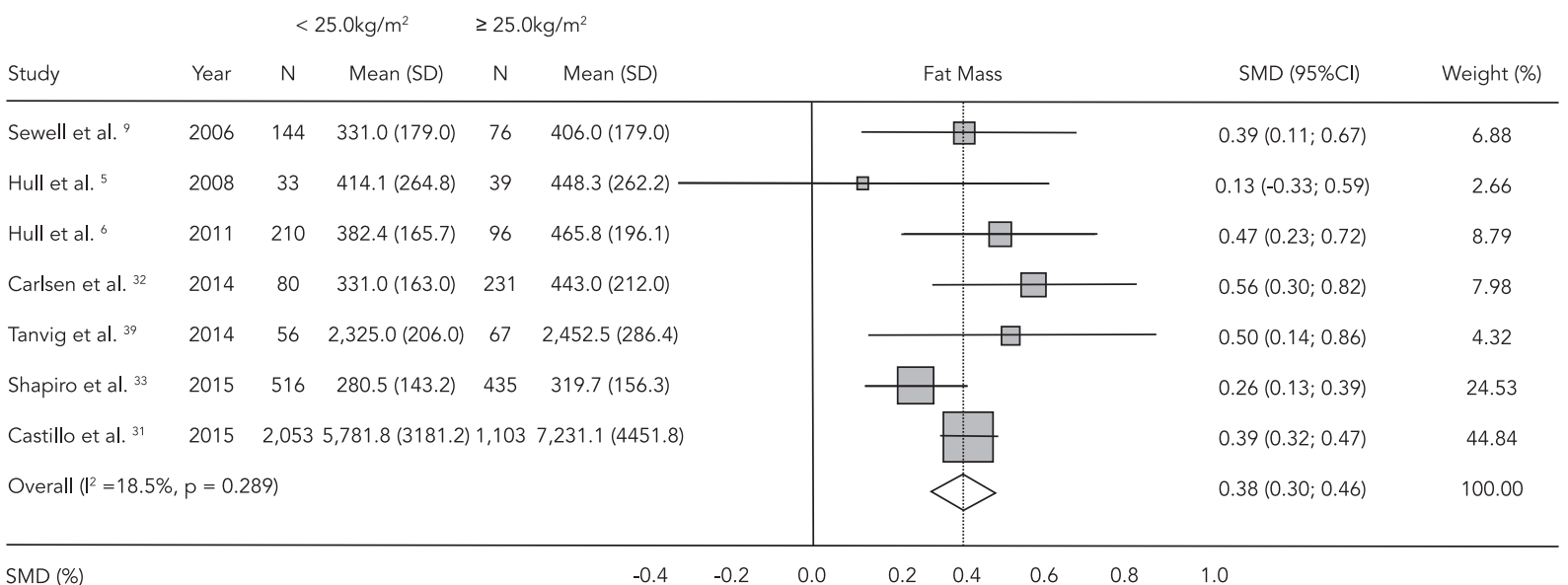

2c) Fat free mass/Maternal pre-pregnancy BMI

$$
<25.0 \mathrm{~kg} / \mathrm{m}^{2} \quad \geq 25.0 \mathrm{~kg} / \mathrm{m}^{2}
$$

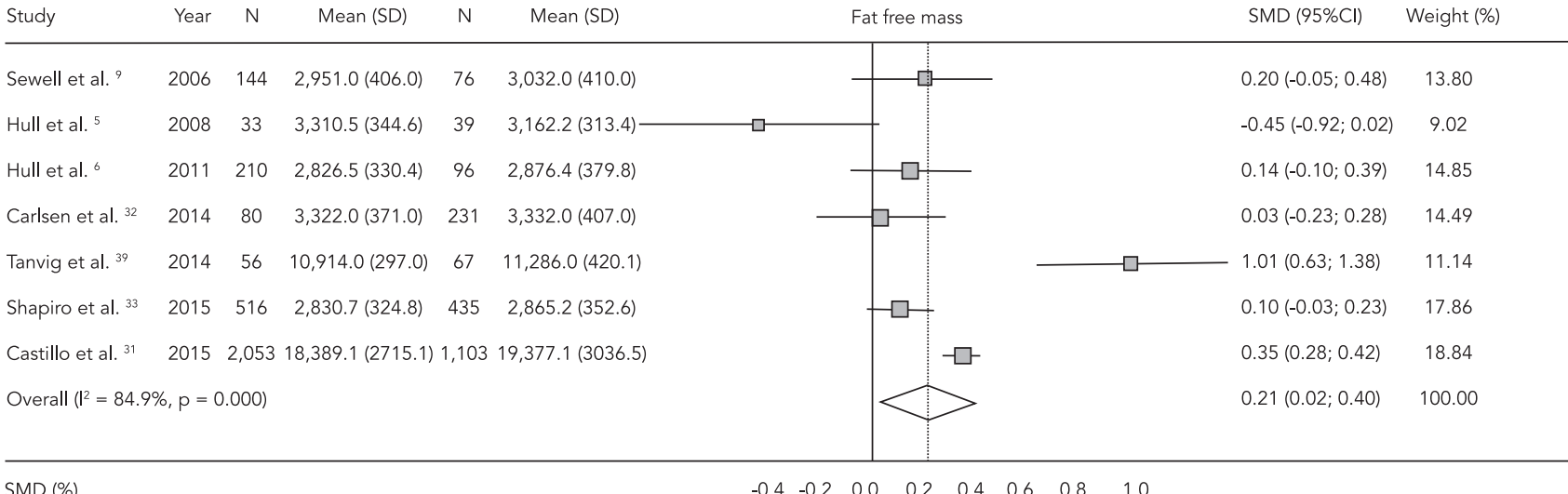

Note: weight are from random effects analysis.

95\% $\mathrm{Cl}$ : 95\% confidence interval; SMD: standard mean difference (\%). 
and adjusted analysis). The joint test for all five covariates provided a $\mathrm{p}$-value $=0.63$, indicating no evidence for an association of at least one of the covariates with the size of the pre-pregnancy effect on offspring fat-free mass. A high residual heterogeneity was maintained in the subgroup analyses with an exception of outcome age measurement: SMD = 0.12 (95\%CI: $-0.03 ; 0.27), \mathrm{I}^{2}=$ $0.00 \%$ in studies with $<1$ week outcome age measurement and SMD = 0.27 (95\%CI: $-0.03 ; 0.57$ ), $\mathrm{I}^{2}=$ $91.2 \%$ in studies with outcome age measurement $\geq 1$ week.

In summary, whilst some studies report a positive association between maternal pre-pregnancy BMI and the body fat of children, the outcomes have been variably assessed in terms of body fat percent, fat mass or FMI. Therefore it is clear that the majority of studies assessed the relationship between pre-pregnancy BMI and the offspring's body composition in terms of childhood adiposity rather than the fat-free component.

\section{Studies that assessed the effect of gestational weight gain}

Ten studies assessed the relationship between gestational weight gain and offspring body composition 6,27,28,29,30,31,41,42,43,44 Four studies $31,41,42,44$ assessed gestational weight gain according to pre-pregnancy BMI and divided it into categories as recommended by the Institute of Medicine of the U.S. National Academy of Sciences 45 . Sewell et al. 29 applied a pre-pregnancy BMI cut-off $\geq 25 \mathrm{~kg} / \mathrm{m}^{2}$. Seven studies assessed gestational weight gain as a continuous variable $6,27,28,30,31,37,42$. Six studies measured body composition in newborns $6,27,29,41,42,44$, one in children under a year in age 43 and five in children from 3-10 years of age 28,30,31,42,43.

\section{- Maternal gestational weight gain and offspring adiposity}

The relationship between gestational weight gain and body fat percent was evaluated in seven studies $6,27,29,31,37,41,44$, fat mass in nine 6,29,32,36,37,39,40,41,43, and FMI in two 28,31 (Table 3). Five studies reported a statistically significant positive effect of gestational weight gain on body fat percent in univariate analysis $6,29,31,41,44$ and three studies $27,31,37$ reported a positive effect between gestational weight gain and body fat percent in the multivariate analysis. In addition, a significant direct association between gestational weight gain and fat mass was found following univariate analysis in four studies 6,31,41,42.

\section{- Maternal gestational weight gain and offspring lean mass or fat-free mass}

The relationship of maternal gestational weight gain and fat-free mass was evaluated in seven studies 6,31,37,41,42,43,44 and between maternal gestational weight gain and LMI or FFMI (fat free mass index) in two 28,31 (Table 3). A positive effect of gestational weight gain on fat-free mass was reported in six studies 6,29,31,41,42,44, and this association was statistically significant in three of them $6,29,31$. Gale et al. ${ }^{28}$ however reported an inverse association of gestational weight gain on LMI.

The crude analyses of the studies that categorized gestational weight gain according to maternal pre-pregnancy BMI showed that children of overweight or obese mothers, with an adequate or excessive gestational weight gain were more likely to have a high fat mass 6,29,31,41,42,43, body fat percent 6,29,31,41,44, and FFMI 28,31 compared to newborns of mothers with normal maternal pre-pregnancy BMI with adequate or excessive weight gain (Table 3 ).

\section{Discussion}

This review suggests that children born from mothers with normal maternal pre-pregnancy BMI have lower fat mass and body fat percent than those born from overweight or obese mothers $27,28,31,32,34,35,36,37,40$. The aggregate measure estimated a significantly small difference in mean fat mass and body fat percent in the first months of life between children of overweight or obese mothers and those from mothers with normal maternal pre-pregnancy BMI. The visual inspection of the forest plot showed that effect size and direction of associations described by Hull et al. ${ }^{5}$ and Tanvig et al. 39 are clearly different. In fact the sample size of the studies can explain such a discrepancy, since both studies have less than 100 participants. There was a significant difference in terms of fat-free mass; random-effects model of meta-analysis shows a significant association of maternal pre-pregnancy BMI and offspring fat mass, body fat percent and fat-free mass. The databases included studies conducted in high and middle income countries, so the meta-analysis lacks information from low income countries populations. An aggregate analysis of gestational weight gain and fat mass, body fat percent or fatfree mass could not be performed.

There are few studies investigating the effect of maternal pre-pregnancy BMI or gestational weight gain on children's body composition as measured by indirect methods. Consequently, 
Maternal gestational weight gain and offspring body composition in childhood.

\begin{tabular}{|c|c|c|c|c|}
\hline Author & Maternal exposure & Offspring outcome & Crude analyses & Adjusted analyses \\
\hline \\
\hline \multicolumn{5}{|l|}{ body fat percent } \\
\hline \multirow[t]{13}{*}{ Hull et al. 6} & Gestational weight gain $(\mathrm{kg})$ & $\begin{array}{c}\text { Body fat \% (percentage point) at 24-48 } \\
\text { hours, mean (SD) }\end{array}$ & $12.3(4.4)$ & - \\
\hline & Pre-gestational weight BMI 18- & Body fat \% (percentage point) at 24-48 & $11.7(4.1)$ & \\
\hline & $24 \mathrm{~kg} / \mathrm{m}^{2} /$ Gestational weight gain & hours, mean (SD) & & \\
\hline & Adequade & & & $11.2(0.5)$ \\
\hline & Excessive & & & $11.8(0.5)$ \\
\hline & Pre-gestational weight BMI 25- & & $13.0(4.7)$ & - \\
\hline & $29 \mathrm{~kg} / \mathrm{m}^{2} /$ Gestational weight gain & & & \\
\hline & Adequade & & - & $9.2(1.1)$ \\
\hline & Excessive & & - & $13.7(0.7)$ \\
\hline & Pre-gestational weight $\mathrm{BMI} \geq$ & & $14.6(4.3)$ & \\
\hline & 30kg/m2/Gestational weight gain & & & \\
\hline & Adequade & & - & $14.6(1.4)$ \\
\hline & Excessive & & - & $14.2(0.8)$ \\
\hline \multirow[t]{4}{*}{ Josefson et al. 41} & Gestational weight gain & & & \\
\hline & Adequate & Body fat \% (percentage point) at 72 & $10.7(2.8)$ & - \\
\hline & & hours, mean (SD) & & \\
\hline & Excessive & & $13.9(3.3)$ & - \\
\hline \multirow[t]{4}{*}{ Henriksson et al. 44} & Gestational weight gain & & & \\
\hline & Inadequate & $\begin{array}{l}\text { Body fat \% (percentage point) at 2-11 } \\
\text { days, mean (SD) }\end{array}$ & $11.1(4.1)$ & - \\
\hline & Adequate & & $11.9(4.1)$ & - \\
\hline & Excessive & & $13.0(3.8)$ & - \\
\hline Sewell et al. 29 & $\begin{array}{l}\text { Gestational weight gain (lb)/Pre- } \\
\text { gestational } \mathrm{BMI} \geq 25 \mathrm{~kg} / \mathrm{m}^{2}\end{array}$ & $\begin{array}{c}\text { Body fat \% (percentage point) at } 72 \\
\text { hours, } r\end{array}$ & 0.4 & - \\
\hline Au et al. 27 & Gestational weight gain $(\mathrm{kg})$ & Difference in body fat $\%, \beta(95 \% \mathrm{Cl})$ & & $0.1(0.1 ; 0.2)$ \\
\hline Starling et al. 37 & Gestational weight gain $(\mathrm{kg})$ & $\begin{array}{l}\text { Body fat } \% \text { (percentage point) at } 3 \text { days, } \\
\qquad \beta(95 \% \mathrm{Cl})\end{array}$ & & $0.6(0.4 ; 0.7)$ \\
\hline \multirow[t]{4}{*}{ Castillo et al. 31} & Gestational weight gain $(\mathrm{kg})$ & $\begin{array}{c}\text { Body fat } \% \text { (percentage ppoint) at } 6 \\
\text { years, } \beta(95 \% \mathrm{Cl})\end{array}$ & $0.16(0.12 ; 0.21)$ & $0.18(0.13 ; 0.23)$ \\
\hline & Insufficient & $\begin{array}{c}\text { Body fat \% (percentage point) at } 6 \text { years, } \\
\text { mean (SD) }\end{array}$ & $22.1(7.4)$ & \\
\hline & Sufficient & & $23.5(7.8)$ & \\
\hline & Excessive & & $25.4(8.5)$ & \\
\hline
\end{tabular}

(continues)

only 20 articles met the inclusion criteria for this review, of which ten assessed the maternal prepregnancy BMI, four the gestational weight gain and six both exposures. No studies with negative results were found. Therefore, publication bias can be present 46 . In addition, studies assessing children's body composition by indirect methods conducted in low-income countries were not found in the literature search.

Several methodological aspects of the studies reviewed require consideration. Firstly, four studies used pre-pregnancy BMI or gestational weight gain information as determined by maternal weight data from medical records. Two studies used maternal weight measurements and four studies relied upon self-reported maternal weight information allowing for recall bias. Indeed, studies that evaluated the accuracy of selfreported maternal weight found that women underestimated their own weight 47 which may result in a miscalculation of pre-pregnancy BMI or gestational weight gain and biased categori- 
Table 3 (continued)

\begin{tabular}{|c|c|c|c|c|}
\hline Author & Maternal exposure & Offspring outcome & Crude analyses & Adjusted analyses \\
\hline \multirow{2}{*}{\multicolumn{5}{|c|}{$\begin{array}{l}\text { Studies that assessed } \\
\text { fat mass }\end{array}$}} \\
\hline & & & & \\
\hline \multirow[t]{11}{*}{ Crozier et al. 42} & Gestational weight gain $(\mathrm{kg})$ & Fat mass $\left(\mathrm{kg}^{2}\right), \beta(95 \% \mathrm{Cl})$ & & \\
\hline & & At birth & $0.1(0.0 ; 0.1)$ & - \\
\hline & & At 4 years & $-0.01(-0.1 ; 0.1)$ & $0.0(-0.1 ; 0.1)$ \\
\hline & & At 6 years & $0.1(-0.0 ; 0.1)$ & $0.1(-0.0 ; 0.1)$ \\
\hline & Gestational weight gain & & & \\
\hline & Inadequate & Fat mass $\left(\mathrm{kg}^{2}\right)$ at birth, $\beta(95 \% \mathrm{Cl})$ & $-0.1(-0.3 ; 0.1)$ & $-0.1(-0.3 ; 0.1)$ \\
\hline & Excessive & & $0.1(-0.0 ; 0.3)$ & $0.2(0.0 ; 0.3)$ \\
\hline & Inadequate & Fata mass $\left(\mathrm{kg}^{2}\right)$ at 4 years, $\beta(95 \% \mathrm{Cl})$ & $0.2(-0.1 ; 0.4)$ & $0.2(-0.1 ; 0.4)$ \\
\hline & Excessive & & $0.2(0.0 ; 0.4)$ & $0.1(-0.0 ; 0.3)$ \\
\hline & Inadequate & Fat mass $\left(\mathrm{kg}^{2}\right)$ at 6 years, $\beta(95 \% \mathrm{Cl})$ & $0.2(-0.1 ; 0.4)$ & $0.2(-0.1 ; 0.4)$ \\
\hline & Excessive & & $0.4(0.2 ; 0.5)$ & $0.3(0.1 ; 0.5)$ \\
\hline \multirow[t]{2}{*}{ Gale et al. 28} & Gestational weight gain $(\mathrm{kg})$, SDS & FMI (SDS) male, $\beta$ (95\%Cl) & $0.1(-0.1 ; 0.3)$ & $0.1(-0.1 ; 0.3)$ \\
\hline & & FMI (SDS) female, $\beta$ (95\% Cl) & $0.01(-0.2 ; 0.2)$ & $0.1(-0.0 ; 0.3)$ \\
\hline \multirow[t]{3}{*}{ Henriksson et al. 44} & Gestational weight gain & & & \\
\hline & Inadequate & Fat mass (g) at $2-11$ days, $\beta(95 \% \mathrm{Cl})$ & $-54(-111 ; 4)$ & $-30(-78 ; 18)$ \\
\hline & Excessive & & $55(10 ; 100)$ & $37(-1 ; 75)$ \\
\hline Perng et al. 30 & $\begin{array}{l}\text { Gestational weight gain BMI per } \\
5 \mathrm{~kg} / \mathrm{m}^{2}\end{array}$ & Fat mass $(\mathrm{kg})$ at $6-10$ years, $\beta(95 \% \mathrm{Cl})$ & & $0.3(0.1 ; 0.5)$ \\
\hline \multirow[t]{13}{*}{ Hull et al. 6} & Gestational weight gain $(\mathrm{kg})$ & Fat mass (g) at $24-48$ hours, mean (SD) & $408.5(179.7)$ & - \\
\hline & Pre-gestational BMI 18-24kg/m²/ & Fat mass (g) at 24-48 hours, mean (SD) & $382.4(165.7)$ & \\
\hline & Gestational weight gain & & & \\
\hline & Adequade & & - & $355.5(20.1)$ \\
\hline & Excessive & & - & $388.90(21.9)$ \\
\hline & Pre-gestational BMI 25-29kg/m²/ & & $450.9(216.3)$ & - \\
\hline & Gestational weight gain & & & \\
\hline & Adequade & & - & $303.6(46.1)$ \\
\hline & Excessive & & - & $484.40(28.8)$ \\
\hline & Pre-gestational BMI $\geq 30 \mathrm{~kg} / \mathrm{m}^{2} /$ & & $489.6(158.7)$ & \\
\hline & Gestational weight gain & & & \\
\hline & Adequade & & & $472.9(56.0)$ \\
\hline & Excessive & & & $486.40(33.5)$ \\
\hline \multirow[t]{3}{*}{ Josefson et al. 41} & Gestational weight gain & & & \\
\hline & Sufficient & Fat mass (g) at 72 hours, mean (SD) & $348(103)$ & - \\
\hline & Excessive & & $525(178)$ & - \\
\hline \multirow[t]{4}{*}{ Henriksson et al. 44} & Gestational weight gain & & & \\
\hline & Inadequate & Fat mass $(\mathrm{g})$ at 2-11 days, mean (SD) & $384(178)$ & - \\
\hline & Sufficient & & $438(186)$ & - \\
\hline & Excessive & & $493(177)$ & - \\
\hline \multirow[t]{3}{*}{ Sewell et al. 29} & Gestational weight gain (lb)/ & Fat mass (g) at 72 hours, $r$ & $0.12 *$ & - \\
\hline & Maternal pre-gestational BMI < & & & \\
\hline & $25 \mathrm{~kg} / \mathrm{m}^{2}$ & & & \\
\hline \multirow[t]{3}{*}{ Estampador et al. 43} & Gestational weight gain & & & \\
\hline & Midpregnancy & Fat mass (z-score) at 4 months, $r(95 \% \mathrm{Cl})$ & $0.4(0.04 ; 0.7)$ * & - \\
\hline & Late-pregnancy & & $-0.02(-0.4 ; 0.7)$ & - \\
\hline Starling et al. 37 & Gestational weight gain (kg) & Fat mass (g) at 3 days, $\beta(95 \% \mathrm{Cl})$ & & $24.0(17.4 ; 30.5)$ \\
\hline
\end{tabular}

(continues) 
Table 3 (continued)

\begin{tabular}{|c|c|c|c|c|}
\hline Author & Maternal exposure & Offspring outcome & Crude analyses & Adjusted analyses \\
\hline \multirow{2}{*}{\multicolumn{5}{|c|}{$\begin{array}{l}\text { Studies that assessed } \\
\text { fat mass }\end{array}$}} \\
\hline & & & & \\
\hline \multirow[t]{9}{*}{ Castillo et al. 31} & Gestational weight gain $(\mathrm{kg})$ & Fat mass $(\mathrm{kg})$ at 6 years, $B(95 \% \mathrm{Cl})$ & $0.08(0.06 ; 0.10)$ & $0.08(0.06 ; 0.11)$ \\
\hline & Gestational weight gain $(\mathrm{kg})$ & $\mathrm{FMI}\left(\mathrm{kg} / \mathrm{m}^{2}\right)$ at 6 years, $\mathrm{B}(95 \% \mathrm{Cl})$ & $0.04(0.03 ; 0.06)$ & $0.05(0.04 ; 0.06)$ \\
\hline & Gestational weight gain & & & \\
\hline & Insufficient & Fat mass $(\mathrm{kg})$ at 6 years, mean (SD) & $5.5(3.1)$ & \\
\hline & Sufficient & & $6.1(3.5)$ & \\
\hline & Excessive & & $7.2(4.3)$ & \\
\hline & Insufficient & $\mathrm{FMI}\left(\mathrm{kg} / \mathrm{m}^{2}\right)$ at 6 years, mean (SD) & $3.8(2.0)$ & \\
\hline & Sufficient & & $4.1(2.1)$ & \\
\hline & Excessive & & $4.7(2.5)$ & \\
\hline \multirow{3}{*}{\multicolumn{5}{|c|}{$\begin{array}{l}\text { Studies that assessed } \\
\text { fat-free mass or lean } \\
\text { mass }\end{array}$}} \\
\hline & & & & \\
\hline & & & & \\
\hline \multirow[t]{11}{*}{ Crozier et al. 42} & Gestational weight gain $(\mathrm{kg})$ & Fat-free mass $(\mathrm{kg}), B(95 \% \mathrm{Cl})$ & & \\
\hline & & at birth & $0.01(-0.03 ; 0.1)$ & - \\
\hline & & at 4 years & $0.01(-0.03 ; 0.1)$ & $0.04(-0.01 ; 0.1)$ \\
\hline & & at 6 years & $-0.01(-0.1 ; 0.04)$ & $-0.01(-0.1 ; 0.1)$ \\
\hline & Gestational weight gain & & & \\
\hline & Inadequate & Fat-free mass $(\mathrm{kg})$ at birth, B $(95 \% \mathrm{Cl})$ & $-0.03(-0.2 ; 0.1)$ & $-0.04(-0.2 ; 0.1)$ \\
\hline & Excessive & & $0.02(-0.1 ; 0.1)$ & $0.1(-0.1 ; 0.2)$ \\
\hline & Inadequate & Fat-free mass $(\mathrm{kg})$ at 4 years, B $(95 \% \mathrm{Cl})$ & $0.1(-0.1 ; 0.2)$ & $0.2(-0.1 ; 0.4)$ \\
\hline & Excessive & & $0.1(-0.1 ; 0.2)$ & $0.1(-0.03 ; 0.3)$ \\
\hline & Inadequate & Fat-free mass $(\mathrm{kg})$ at 6 years, B $(95 \% \mathrm{Cl})$ & $0.1(-0.1 ; 0.2)$ & $0.03(-0.1 ; 0.2)$ \\
\hline & Excessive & & $0.1(-0.1 ; 0.2)$ & $0.03(-0.1 ; 0.2)$ \\
\hline \multirow[t]{3}{*}{ Gale et al. 28} & Gestational weight gain $(\mathrm{kg}), \mathrm{SDS}$ & LMI (SDS) & & \\
\hline & & Male B (95\%Cl) & $-0.1(-0.2 ; 0.1)$ & $-0.1(-0.2 ; 0.1)$ \\
\hline & & Female B $(95 \% \mathrm{Cl})$ & $-0.02(-0.2 ; 0.2)$ & $0.01(-0.2 ; 0.2)$ \\
\hline \multirow[t]{3}{*}{ Henriksson et al. 44} & Gestational weight gain & & & \\
\hline & Inadequate & Fat-free mass (g) at $2-11$ days, $B(95 \% \mathrm{Cl})$ & $-152(-263 ;-41)$ & $-13(-77 ; 51)$ \\
\hline & Excessive & & $101(15 ; 187)$ & $39(-12 ; 89)$ \\
\hline \multirow[t]{13}{*}{ Hull et al. 6} & Gestational weight gain $(\mathrm{kg})$ & $\begin{array}{l}\text { Fat-free mass (g) at 24-48 hours, mean } \\
\qquad(\mathrm{SD})\end{array}$ & $2,842.2(346.8)$ & - \\
\hline & Pre-gestational BMI $18-24 \mathrm{~kg} / \mathrm{m}^{2} /$ & Fat-free mass (g) at 24-48 hours, mean & $2,826.5(330.4)$ & - \\
\hline & Gestational weight gain & $(\mathrm{SD})$ & & \\
\hline & Adequade & & - & $2758.7(32.8)$ \\
\hline & Excessive & & - & $2856.60(35.7)$ \\
\hline & Pre-gestational BMI $25-29 \mathrm{~kg} / \mathrm{m}^{2} /$ & & $2,903.3(407.4)$ & \\
\hline & Gestational weight gain & & & \\
\hline & Adequade & & & $2839.7(75.3)$ \\
\hline & Excessive & & & $2968.80(47.0)$ \\
\hline & Pre-gestational BMI 30kg/m²/ & & $2,833.6(332.1)$ & \\
\hline & Gestational weight gain & & & \\
\hline & Adequade & & - & $2792.5(91.5)$ \\
\hline & Excessive & & - & $2913.20(54.7)$ \\
\hline \multirow[t]{3}{*}{ Josefson et al. 41} & Gestational weight gain & & & \\
\hline & Sufficient & Fat-free mass (g), at 72 hours, mean (SD) & $2,898(327)$ & - \\
\hline & Excessive & & $3,174(415)$ & - \\
\hline
\end{tabular}

(continues) 
Table 3 (continued)

\begin{tabular}{|c|c|c|c|c|}
\hline Author & Maternal exposure & Offspring outcome & Crude analyses & Adjusted analyses \\
\hline \multicolumn{5}{|l|}{ Studies that assessed } \\
\hline \multicolumn{5}{|l|}{ fat-free mass or lean } \\
\hline \multicolumn{5}{|l|}{ mass } \\
\hline \multirow[t]{5}{*}{ Henriksson et al. 44} & Gestational weight gain & & & \\
\hline & Inadequate & Fat-free mass (g), at 2-11 days, mean & $2,986(343)$ & - \\
\hline & & $(\mathrm{SD})$ & & \\
\hline & Sufficient & & $3,138(328)$ & - \\
\hline & Excessive & & $3,239(364)$ & - \\
\hline \multirow[t]{4}{*}{ Sewell et al. 29} & Gestational weight gain (lb)/ & & & \\
\hline & Maternal pre-gestational BMI & & & \\
\hline & $<25 \mathrm{~kg} / \mathrm{m}^{2}$ & Fat-free mass (g) at 72 hours, $r$ & 0.20 & 0.19 \\
\hline & $\geq 25 \mathrm{~kg} / \mathrm{m}^{2}$ & & 0.08 & - \\
\hline \multirow[t]{4}{*}{ Estampador et al. 43} & Gestational weight gain & & & \\
\hline & Mid-pregnancy & Fat-free mass (z-score) at 4 months, $r$ & $0.1(-0.3 ; 0.4)$ * & \\
\hline & & $(95 \% \mathrm{Cl})$ & & 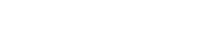 \\
\hline & Late-pregnancy & & $0.4(0.01 ; 0.6)$ & - \\
\hline Starting et al. 37 & Gestational weight gain (kg) & Fat-free mass $(\mathrm{g})$ at 3 days, B $(95 \% \mathrm{Cl})$ & & $34.0(21.4 ; 46.6)$ \\
\hline \multicolumn{5}{|l|}{ Castillo et al. 31} \\
\hline & Gestational weight gain $(\mathrm{kg})$ & Fat-free mass $(\mathrm{kg})$ at 6 years, B $(95 \% \mathrm{Cl})$ & $0.06(0.04 ; 0.07)$ & $0.04(0.02 ; 0.05)$ \\
\hline & Gestational weight gain (kg) & FFMI $\left(\mathrm{kg} / \mathrm{m}^{2}\right)$ at 6 years, $B(95 \% \mathrm{Cl})$ & $0.01(0.006 ; 0.02)$ & $0.01(0.01 ; 0.02)$ \\
\hline & Insufficient & & $18.2(2.7)$ & \\
\hline & Sufficient & & $18.7(2.8)$ & \\
\hline & Excessive & Fat-free mass $(\mathrm{kg})$ at 6 years, mean (SD) & $19.3(3.0)$ & \\
\hline & Insufficient & & $12.6(1.1)$ & \\
\hline & Sufficient & & $12.7(1.1)$ & \\
\hline & Excessive & FFMI $\left(\mathrm{kg} / \mathrm{m}^{2}\right)$ at 6 years, mean (SD) & $13.0(1.2)$ & \\
\hline
\end{tabular}

95\% Cl: 95\% confidence interval; ß: beta coefficient; BMI: body mass index; FFMI: fat-free mass index; FMI: fat mass index (kg/m²); LBMI: lean body mass index $\left(\mathrm{kg} / \mathrm{m}^{2}\right) ; r=$ correlation; SD: standard deviation; SDS: standard deviation score; SE: standard error.

Note: in bold p-value statistically significant.

* Spearman partial correlation coefficients and $95 \% \mathrm{Cl}$.

zation of pregnant women, indicating exposure bias 46,48 .

Secondly, the recent publication period of the 20 articles (between 2005 and 2015) is probably due to the outcomes definition employed. Previously validated studies of DEXA and ADP are available in the literature from 1980 to 1990, respectively. These are however, costly equipment that require operation by trained personnel and constant maintenance.

Thirdly, a lack of homogeneity in maternal pre-pregnancy BMI categorization in two or more groups using different cutoffs was found in the literature search 5,6,27,29,32,33,35,39,40. Lack of definition of cutoff points 34,38 was also observed. These may have unpredictably underestimated or overestimated the effect of maternal pre-pregnancy BMI and gestational weight gain on offspring body composition.
Fourth, confounding variables showed a wide heterogeneity. Child's age, gender and breastfeeding were not investigated as potential effect modifiers, and some studies included these variables in the multivariate analysis. A single study included an analysis stratified by gender 28 and two others investigated current children's weight as an effect modifier 28,34.

Fifth, few studies included socioeconomic characteristics as confounding variables in the adjusted analysis. However, the effect of environmental factors was present in the four studies that adjusted for family income, education level, race, and maternal smoking during pregnancy $31,34,35,38$ strengthening the association (negative confounding) 49 . The history of maternal smoking during pregnancy was considered in the adjusted analysis of eight studies 28,29,31,32,35,38,42,44. The maternal smoking during pregnancy is a 
well-known cause of growth restriction and low birth-weight and recent studies also suggested that pre-natal smoking compromises infant growth 2,21,49. The mechanism underlying this association is unknown. Some speculate that this association may result from confounding. Mothers who smoke during pregnancy may provide a different social environment for the offspring than mothers who do not smoke 21,42.

On the other hand, the inclusion of current child characteristics such as height, weight, conditional weight gain or birth weight as confounding variables seems to be inappropriate $28,34,35,36,38$. These variables would more likely have a mediating rather than a confounding effect on the relationship between pre-pregnancy BMI and gestational weight gain with offspring body composition 48,50 .

Different studies have reported a positive association between maternal pre-pregnancy BMI and the weight of children born at full term $11,13,29$. Since the first study on the relationship between children's body composition and maternal pre-pregnancy BMI or gestational weight gain as the exposure published in 2001 7, evidence shows that the incidence of newborns weighing more than $4,000 \mathrm{~g}$ at birth increases as pre-pregnancy BMI shifts from normal to obesity 51 . Due to the availability of indirect methods for body composition the fat mass and fat-free mass arose as new components of birth weight. Studies 5,6,29 have reported that in full-term newborns, fat-free mass accounts for $70-87 \%$ of the variation in birth weight. In the relationship of pre-pregnancy BMI with children's body composition born at full-term to overweight/ obese mothers, fat-free mass accounts for 61$75 \%$ variation on birth weight while it accounts for $74-89 \%$ variation in those who are born from mothers with normal pre-pregnancy BMI 5,6,29. Thus, an increase in birth weight that is associated with maternal pre-pregnancy BMI may be primarily attributed to an increase in FM rather than fat-free mass 29 .

Gale et al. 28 and Castillo et al. 31 were the studies that provided data on FMI, FFMI and LMI. Children can have different percentages of fat mass due to different absolute amounts of fat mass but equivalent amounts of fat-free mass or different absolute amounts of fat-free mass and same amounts of fat mass. Because of these relations the normalization of fat mass and lean mass measures for squared height $\left(\mathrm{kg} / \mathrm{m}^{2}\right)$ is recommended to improve the sensitivity to detect changes in body composition measurements 23 .

Fetal over-nutrition hypothesis is one mechanism that has been suggested to explain the relationship between maternal pre-pregnancy BMI and gestational body composition and offspring body composition. So the relationship between maternal gestational obesity and offspring obesity could be explained by the effect of the exposure to high maternal plasma glucose, free fatty acid and amino acids concentration during pregnancy carrying lifelong changes in fetal appetite, neuroendocrine function or energy metabolism and an increased risk for metabolic and cardiovascular diseases after birth 1,4 .

Studies on intergenerational heritability with family members suggest a role of genetic factors in determining individual differences in adiposity 52 . Therefore, a variant of the fat mass and obesity associated gene in homozygous individuals appears to increase the risk of obesity, when compared to individuals without the allele. The same is seen for fat mass in children 53. Lawlor et al. 38 investigated the effect of pre-pregnancy BMI on adiposity in children aged 9-11 years and found that the effect of maternal pre-pregnancy BMI and of the fat mass and obesity associated gene on children FM lost statistical significance after adjusting for child's fat mass and obesity associated as an instrumental variable. The mechanisms involved in the association of the fat mass and obesity associated gene and the high adiposity are still unclear.

From a perspective of disease prevention and health promotion, maternal obesity is a potentially preventable cause to reduce the disease and mortality burden in childhood. Yet prenatal counseling should be cautious when advising overweight or obese women to optimize their weight before pregnancy. McDonald et al. 49 argued that maternal overweight/obesity may have a beneficial effect on the survival of children born with low birth weight in low-income countries despite the increased risk of preterm birth in this group.

To our knowledge, this is the first review investigating children's body composition assessed by indirect methods associated with maternal pre-pregnancy BMI and gestational weight gain and highlights the importance of new studies with appropriate methodology. Given the small number of publications with a number of methodological limitations found in this systematic review and meta-analysis, further investigations are required to evidence causality criteria and to strengthen the association between BMI and gestational weight gain and children's body composition. Therefore, fetal growth monitoring during pregnancy with the assessment of pre-gestational and gestational maternal body composition (by other more accurate methods than maternal weight) are required as well as the use of maternal and children's genetic instrumental variables. 


\section{Resumo}

Esta revisão avaliou a associação entre o índice de massa corporal (IMC) pré-gestacional elou ganho de peso gestacional com a composição corporal dos filhos na infância. Uma revisão sistemática foi realizada. Estudos de coorte, caso-controle e ensaios randomizados, que determinaram a composição corporal dos filhos mediante métodos indiretos, foram incluídos. Meta-análises do efeito do IMC pré-gestacional sobre a massa livre de gordura, percentual de gordura corporal e massa gorda foram realizados. Incluiu-se 18 estudos, a maioria dos quais reportou associação positiva entre o IMC materno pré-gestacional e adiposidade dos filhos. As diferenças de médias padronizadas de percentual de gordura corporal, massa gorda e massa livre de gordura entre filhos de mulheres com IMC pré-gestacional normal em comparação com os de mulheres com sobrepeso/obesidade foram: 0,31 pontos percentuais (IC95\%: 0, 19; 0,42), 0,38kg (IC95\%: 0,26; 0,50) e 0,18kg (IC95\%: -0,07; 0,42), respectivamente. Esta evidência sugere que o sobrepeso materno pré-gestacional está associado com a elevada adiposidade em seus filhos.

Obesidade; Gravidez; Composição Corporal; Adiposidade

\section{Contributors}

H. Castillo-Laura. and I. S. Santos conceived the paper, conducted the analysis and wrote the manuscript. L. C. M. Quadros and A. Matijasevich contributed to the design of the study. All authors revised and approved the final version of the paper for publication.

\section{Acknowledgments}

We would like to thank the following organizations for their support in the production of knowledge for this study: Postgraduate Program in Epidemiology at the Pelotas Federal University and Wellcome Trust.

\section{References}

1. Symonds ME, Mendez MA, Meltzer HM, Koletzko B, Godfrey K, Forsyth S, et al. Early life nutritional programming of obesity: mother-child cohort studies. Ann Nutr Metab 2013; 62:137-45.

2. Kramer MS. Determinants of low birth weight: methodological assessment and meta-analysis. Ann Nutr Metab 1987; 65:663-737.

3. Schlussel MM, Souza EB, Reichenheim ME, Kac G. Physical activity during pregnancy and maternalchild health outcomes: a systematic literature review. Cad Saúde Pública 2008; 24 Suppl 4:531-44

4. Armitage JA, Poston L, Taylor PD. Developmental origins of obesity and the metabolic syndrome: the role of maternal obesity. Front Horm Res 2008; 36:73-84.

5. Hull HR, Dinger MK, Knehans AW, Thompson DM, Fields DA. Impact of maternal body mass index on neonate birthweight and body composition. Am J Obstet Gynecol 2008; 198:416.e1-6.

6. Hull HR, Thornton JC, Ji Y, Paley C, Rosenn B, Mathews P, et al. Higher infant body fat with excessive gestational weight gain in overweight women. Am J Obstet Gynecol 2011; 205:211.e1-7.

7. Laitinen J, Power C, Jarvelin MR. Family social class, maternal body mass index, childhood body mass index, and age at menarche as predictors of adult obesity. Am J Clin Nutr 2001; 74:287-94.

8. Tequeanes AL, Gigante DP, Assuncao MC, Chica DA, Horta BL. Maternal anthropometry is associated with the body mass index and waist:height ratio of offspring at 23 years of age. J Nutr 2009; 139:750-4

9. Ehrenberg HM, Dierker L, Milluzzi C, Mercer BM. Prevalence of maternal obesity in an urban center. Am J Obstet Gynecol 2002; 187:1189-93.

10. Lu GC, Rouse DJ, DuBard M, Cliver S, Kimberlin D, Hauth JC. The effect of the increasing prevalence of maternal obesity on perinatal morbidity. Am J Obstet Gynecol 2001; 185:845-9.

11. Ehrenberg HM, Mercer BM, Catalano PM. The influence of obesity and diabetes on the prevalence of macrosomia. Am J Obstet Gynecol 2004; 191:964-8

12. Jensen DM, Damm P, Sorensen B, Molsted-Pedersen L, Westergaard JG, Ovesen P, et al. Pregnancy outcome and prepregnancy body mass index in 2459 glucose-tolerant Danish women. Am J Obstet Gynecol 2003; 189:239-44.

13. Surkan PJ, Hsieh CC, Johansson AL, Dickman PW, Cnattingius S. Reasons for increasing trends in large for gestational age births. Obstet Gynecol 2004; 104:720-6.

14. Ogden CL, Carroll MD, Curtin LR, McDowell MA, Tabak CJ, Flegal KM. Prevalence of overweight and obesity in the United States, 1999-2004. JAMA 2006; 295:1549-55.

15. Boney CM, Verma A, Tucker R, Vohr BR. Metabolic syndrome in childhood: association with birth weight, maternal obesity, and gestational diabetes mellitus. Pediatrics 2005; 115:290-6.

16. Pettitt DJ, Baird HR, Aleck KA, Bennett PH, Knowler WC. Excessive obesity in offspring of Pima Indian women with diabetes during pregnancy. N Engl J Med 1983; 308:242-5. 
17. Mamun AA, O'Callaghan M, Callaway L, Williams G, Najman J, Lawlor DA. Associations of gestational weight gain with offspring body mass index and blood pressure at 21 years of age: evidence from a birth cohort study. Circulation 2009; 119:1720-7.

18. Moreira P, Padez C, Mourão-Carvalhal I, Rosado V. Maternal weight gain during pregnancy and overweight in Portuguese children. Int J Obes (Lond) 2007; 31:608-14.

19. Oken E, Taveras EM, Kleinman KP, Rich-Edwards JW, Gillman MW. Gestational weight gain and child adiposity at age 3 years. Am J Obstet Gynecol 2007; 196:322.e1-8.

20. Catalano PM, Drago NM, Amini SB. Maternal carbohydrate metabolism and its relationship to fetal growth and body composition. Am J Obstet Gynecol 1995; 172:1464-70.

21. Koupil I, Toivanen P. Social and early-life determinants of overweight and obesity in 18-year-old Swedish men. Int J Obes (Lond) 2008; 32:73-81.

22. Stuebe AM, Forman MR, Michels KB. Maternalrecalled gestational weight gain, pre-pregnancy body mass index, and obesity in the daughter. Int J Obes (Lond) 2009; 33:743-52.

23. Wells JC, Fuller NJ, Dewit O, Fewtrell MS, Elia M, Cole TJ. Four-component model of body composition in children: density and hydration of fat-free mass and comparison with simpler models. Am J Clin Nutr 1999; 69:904-12.

24. Wells JC, Fewtrell MS. Measuring body composition. Arch Dis Child 2006; 91:612-7.

25. Moher D, Liberati A, Tetzlaff J, Altman DG, Group P. Preferred reporting items for systematic reviews and meta-analyses: the PRISMA statement. PLoS Med 2009; 6:e1000097.

26. Downs SH, Black N. The feasibility of creating a checklist for the assessment of the methodological quality both of randomised and non-randomised studies of health care interventions. J Epidemiol Community Health 1998; 52:377-84.

27. Au CP, Raynes-Greenow CH, Turner RM, Carberry AE, Jeffery H. Fetal and maternal factors associated with neonatal adiposity as measured by air displacement plethysmography: a large cross-sectional study. Early Hum Dev 2013; 89:839-43.

28. Gale CR, Javaid MK, Robinson SM, Law CM, Godfrey KM, Cooper C. Maternal size in pregnancy and body composition in children. J Clin Endocrinol Metab 2007; 92:3904-11.

29. Sewell MF, Huston-Presley L, Super DM, Catalano P. Increased neonatal fat mass, not lean body mass, is associated with maternal obesity. Am J Obstet Gynecol 2006; 195:1100-3.

30. Perng W, Gillman MW, Mantzoros CS, Oken E. A prospective study of maternal prenatal weight and offspring cardiometabolic health in midchildhood. Ann Epidemiol 2014; 24:793-800.

31. Castillo H, Santos IS, Matijasevich A. Relationship between maternal pre-pregnancy body mass index, gestational weight gain and childhood fatness at 6-7 years by air displacement plethysmography. Matern Child Nutr 2015; 11:606-17.
32. Carlsen EM, Renault KM, Norgaard K, Nilas L, Jensen JE, Hyldstrup L, et al. Newborn regional body composition is influenced by maternal obesity, gestational weight gain and the birthweight standard score. Acta Paediatr 2014; 103:939-45.

33. Shapiro AL, Schmiege SJ, Brinton JT, Glueck D, Crume TL, Friedman JE, et al. Testing the fuel-mediated hypothesis: maternal insulin resistance and glucose mediate the association between maternal and neonatal adiposity, the Healthy Start study. Diabetologia 2015; 58:937-41.

34. Berkowitz RI, Stallings VA, Maislin G, Stunkard AJ. Growth of children at high risk of obesity during the first $6 \mathrm{y}$ of life: implications for prevention. Am J Clin Nutr 2005; 81:140-6.

35. Burdette HL, Whitaker RC, Hall WC, Daniels SR. Maternal infant-feeding style and children's adiposity at 5 years of age. Arch Pediatr Adolesc Med 2006; 160:513-20.

36. Andres A, Hull HR, Shankar K, Casey PH, Cleves MA, Badger TM. Longitudinal body composition of children born to mothers with normal weight, overweight, and obesity. Obesity 2015; 23:1252-8.

37. Starling AP, Brinton JT, Glueck DH, Shapiro AL, Harrod CS, Lynch AM, et al. Associations of maternal BMI and gestational weight gain with neonatal adiposity in the Healthy Start study. Am J Clin Nutr 2015; 101:302-9.

38. Lawlor DA, Timpson NJ, Harbord RM, Leary S, Ness A, McCarthy MI, et al. Exploring the developmental overnutrition hypothesis using parentaloffspring associations and FTO as an instrumental variable. PLoS Med 2008; 5:33.

39. Tanvig M, Vinter CA, Jorgensen JS, Wehberg S, Ovesen PG, Lamont RF, et al. Anthropometrics and body composition by dual energy X-ray in children of obese women: a follow-up of a randomized controlled trial (the Lifestyle in Pregnancy and Offspring [LiPO] study). PLoS One 2014; 9:89590.

40. Pereira-da-Silva L, Cabo C, Moreira AC, Virella D, Guerra T, Camoes T, et al. The adjusted effect of maternal body mass index, energy and macronutrient intakes during pregnancy, and gestational weight gain on body composition of full-term neonates. Am J Perinatol 2014; 31:875-82.

41. Josefson JL, Hoffmann JA, Metzger BE. Excessive weight gain in women with a normal pre-pregnancy BMI is associated with increased neonatal adiposity. Pediatr Obes 2013; 8:e33-6.

42. Crozier SR, Inskip HM, Godfrey KM, Cooper C, Harvey NC, Cole ZA, et al. Weight gain in pregnancy and childhood body composition: findings from the Southampton Women's Survey. Am J Clin Nutr 2010; 91:1745-51.

43. Estampador AC, Pomeroy J, Renstrom F, Nelson SM, Mogren I, Persson M, et al. Infant body composition and adipokine concentrations in relation to maternal gestational weight gain. Diabetes Care 2014; 37:1432-8. 
44. Henriksson P, Eriksson B, Forsum E, Lof M. Gestational weight gain according to Institute of Medicine recommendations in relation to infant size and body composition. Pediatr Obes 2015; 10 : 388-94.

45. Institute of Medicine; National Research Council. Weight gain during pregnancy: reexamining the Guidelines. Washington DC: National Academies Press; 2009.

46. Grimes DA, Schulz KF. Bias and causal associations in observational research. Lancet 2002; 359: 248-52.

47. Engstrom JL, Paterson SA, Doherty A, Trabulsi M, Speer KL. Accuracy of self-reported height and weight in women: an integrative review of the literature. J Midwifery Womens Health 2003; 48 : 338-45.

48. Greenland S, Robins JM. Confounding and misclassification. Am J Epidemiol 1985; 122:495-506.

49. McDonald SD, Han Z, Mulla S, Beyene J; Knowledge Synthesis Group. Overweight and obesity in mothers and risk of preterm birth and low birth weight infants: systematic review and meta-analyses. BMJ 2010; 341:c3428.
50. Christenfeld NJ, Sloan RP, Carroll D, Greenland S. Risk factors, confounding, and the illusion of statistical control. Psychosom Med 2004; 66:868-75.

51. Weiss JL, Malone FD, Emig D, Ball RH, Nyberg DA, Comstock CH, et al. Obesity, obstetric complications and cesarean delivery rate: a populationbased screening study. Am J Obstet Gynecol 2004; 190:1091-7.

52. Maes HH, Neale MC, Eaves LJ. Genetic and environmental factors in relative body weight and human adiposity. Behav Genet 1997; 27:325-51.

53. Frayling TM, Timpson NJ, Weedon MN, Zeggini E, Freathy RM, Lindgren CM, et al. A common variant in the FTO gene is associated with body mass index and predisposes to childhood and adult obesity. Science 2007; 316:889-94.

Submitted on 28/Oct/2014

Final version resubmitted on $08 / \mathrm{Jul} / 2015$

Approved on 23/Jul/2015 